\title{
Modeling and solving cloud service purchasing in multi-cloud environments
}

\author{
Leonard Heilig a,*, Eduardo Lalla-Ruiz ${ }^{\mathrm{b}}$, Stefan Voßa \\ a Institute of Information Systems (IWI), University of Hamburg, Germany \\ ${ }^{\mathrm{b}}$ Department of Industrial Engineering and Business Information Systems, University of Twente, Enschede, Netherlands
}

\section{A R T I C L E I N F O}

\section{Article history:}

Received 30 January 2019

Revised 9 December 2019

Accepted 26 December 2019

Available online 3 January 2020

\section{Keywords:}

Decision support systems

Cloud computing

Multi-cloud

Cloud brokerage

Adaptive large neighborhood search

Microservices

\begin{abstract}
A B S T R A C T
Nowadays, the range of cloud services offered by cloud providers varies sharply and poses a challenge for cloud consumers aiming for the most cost-effective and compliant solutions, especially when operating highly scalable microservice architectures. A remedy can be provided by a cloud brokerage intelligent mechanism selecting cloud services across multiple clouds on behalf of consumers by considering individual goals and requirements. In this paper, we present the Cloud Service Purchasing Problem (CSPP) which aims to minimize costs while incorporating specific consumer and application task requirements. To solve this problem, we propose a mixed-integer programming model and two large neighborhood search approaches. Using well-defined problem instances incorporating data from real cloud providers, we conduct several computational experiments to evaluate the performance of the proposed algorithms. They exhibit a competitive performance providing solutions for all scenarios within short computational times. Finally, the significance of this problem and decision support approaches is analyzed by comparing usage patterns with respect to different nowadays cloud providers and offered virtual machine types for various scenarios.
\end{abstract}

(c) 2020 Elsevier Ltd. All rights reserved.

\section{Introduction}

With the advent of cloud computing, organizations and individuals are able to flexibly consume a variety of information technology (IT) services from cloud marketplaces according to their current needs. Infrastructure as a Service (IaaS) represents one of the dominant forms of cloud computing, allowing cloud consumers to provide virtual computing resources in an on-demand fashion. IDC (2017) predicts an increase of worldwide IaaS spendings by a five-year compound annual growth rate of $30.1 \%$ until 2020. While IaaS provides managed and virtualized IT infrastructure components (e.g., servers, storage, networking), consumers can also use Platform as a Service (PaaS) for developing and hosting software applications without managing virtual infrastructure components, as well as ready-to-use web applications in form of Software as a Service (SaaS). For a detailed introduction to cloud computing, the reader is referred to (Armbrust et al., 2010; Buyya, Yeo, Venugopal, Broberg, \& Brandic, 2009; Heilig \& Voß, 2014a).

Nowadays, more and more companies move their traditional IT architectures into cloud environments. Instead of building mono-

\footnotetext{
* Corresponding author.

E-mail addresses: leonard.heilig@uni-hamburg.de (L. Heilig), e.a.lalla@utwente.nl (E. Lalla-Ruiz), stefan.voss@uni-hamburg.de (S. Voß).
}

lithic systems, many companies nowadays build microservice architectures, where application services are wrapped in a container (e.g., Docker) and hosted on a virtual machine provided by clouds as IaaS. By this, every application service becomes highly scalable, but also requires certain computing resources defined by the virtual machine type. While cloud providers offer almost unlimited computing resources, the range of offered virtual machine types, differing in computing capacities and prices per hour, is continuously growing and leads to an agony of choice. This implies a high complexity when decision makers have to determine an efficient cloud infrastructure configuration for hosting a large set of application tasks (i.e., microservices hosted in containers) with different requirements, including resource and governance requirements, as recently reported in one of the largest global IT leadership surveys (KPMG, 2017, p. 6). In contrast to traditional IT outsourcing, public clouds offer these services for an on-demand use with flexible pricing schemes and scaling options thus enabling drastic reduction in capital and operation expenses for consumers. Given such complexities, it becomes nearly impossible to select the right resources, satisfying the applications requirements, without any decision support. Having intelligent approaches and systems helping assigning the right resources to the application by minimizing a given objective function is therefore required. After defining an appropriate set up, another advancement of clouds is the ease of 
consumption: using cloud services does not require in-person interactions (Vaquero, Rodero-Merino, Caceres, \& Lindner, 2009) so that it only takes seconds to minutes from the initial request to the final deployment.

However, cloud consumers need to address certain outsourcing risks coming along with the adoption of cloud services, such as concerning the risk of shadow-IT, loss of control and transparency, security and business continuity (see, e.g., COSO, 2012). For instance, it is crucial to consider the deployment region as it has a significant impact on data protection regulations and the place of jurisdiction (Subashini \& Kavitha, 2011). In addition, the innovative features of cloud computing (see, e.g., Sultan \& van de Bunt-Kokhuis, 2012), along with a high concentration of expertise at major cloud providers (Chen, Paxson, \& Katz, 2010), can help consumers to improve the security and availability of cloud-based infrastructures and applications (e.g., cross-region redundancy, auto scaling, etc.; see, e.g., Zissis \& Lekkas, 2012). Moreover, there are many concerns about the potential dependency on one cloud provider. While many consumers are locked to a single cloud provider due to interoperability issues, the concepts of cloud service brokerage (e.g., Buyya et al., 2009) and multi-cloud environments (e.g., Rochwerger et al., 2009) have been intensively discussed in academia in recent years. Moreover, automated deployment processes support the technical integration, configuration, and use of IaaS services from multiple cloud providers (Heilig, Voß, \& Wulfken, 2015).

According to Gartner (2016) and NIST (National Institute of Standards and Technology; Liu et al., 2011), cloud service brokerage adds value to one or more cloud services by mediating between one or more consumers and cloud providers in order to govern and manage the use, performance, and delivery of cloud services on behalf of cloud consumers. The potential benefits of cloud brokerage attract global IT players like Dell, launching programs to create brokerage services and marketplaces for multi-cloud environments (Chanthadavong, 2014). One of the core elements for offering brokerage services lies in the selection and delivery of cloud services based on consumer and application task requirements. In this regard, one of the main goals of cloud brokers is to address individual governance policies and gain arbitrage profits by taking advantage of price and feature differences among a wide range of cloud providers and services (Liu et al., 2011), further leading to fierce competition among cloud providers (Püschel, Schryen, Hristova, \& Neumann, 2015). While brokerage schemes involving a third-party may lead to typical principal-agent problems due to information asymmetries, we primarily focus on decision support mechanisms that help consumers in better selecting and utilizing virtual machines in multi-cloud environments by considering specific objectives and constraints, such as the ones regarding the overall costs and deployment region, respectively. Especially the ease of consumption and flexible use of cloud services pose new challenges for organizations, such as related to shadow IT, governance processes, business strategies, and security (Andriole, 2015). Both industry and research would largely benefit from a more efficient utilization of cloud services, specifically areas related to topics like microservices, big data, internet of things, and escience (Hoffa et al., 2008).

The previous discussion leads to the development of mathematical models and algorithms for supporting cloud brokerage when selecting and purchasing appropriate configurations of cloud services from multiple cloud providers on behalf of one or more consumers. In this regard, the objective is to reduce costs and improve the overall performance in terms of specific consumer and application task requirements as well as regarding general IT governance requirements of individual organizations. As there are several manifestations and options of those cloud services, there are many potentials of optimizing the consumption of cloud services.
In the field of cloud computing, several approaches have been presented to address similar problems involving decision-making (for a scientometric analysis, the interested reader is referred to Heilig \& Voß, 2014b). However, as explained in the related-works section, those solutions are not fully applicable as important aspects are not considered, such as aspects that strongly influence the overall deployment and operating costs, like the selected deployment region and operating system.

Our work adds to the literature by presenting and assessing a novel brokerage mechanism for supporting decisions in automated deployment processes regarding the use of computing services in the form of virtual machine types in a multi-cloud environment. The main contributions of this paper are stated as follows.

- We provide a mathematical formulation to model the Cloud Service Purchasing Problem (CSPP) for multi-cloud environments in order to cover important aspects not yet considered in the literature.

- We propose Large Neighborhood Search (LNS) and Adaptive Large Neighborhood Search (ALNS) metaheuristics to address decision making requirements as previously discussed with practitioners (see, e.g. Heilig, Lalla-Ruiz, \& Voß, 2016; Heilig et al., 2015).

- To analyze different scenarios, a set of rich and well-defined problem instances is created. The problem instances represent different types of application tasks and incorporate real data of IaaS compute services offered by the three leading cloud providers, namely Amazon Web Services (AWS), Google, and Microsoft.

- In an extensive computational study, the proposed solution approaches are evaluated in terms of solution quality and computational time based on a determined parameter setting. Additionally, the impact of sharing virtual machines among the applications is assessed. We further investigate the results from a business perspective by comparing cloud providers and analyzing usage and sharing patterns based on various scenarios with standard, memory-intensive, and compute-intensive applications. Thereby, we unveil differences between cloud providers and important aspects regarding the selection and sharing of virtual machine types.

The remainder of this paper is organized as follows. Section 2 provides a literature overview on related works. A mathematical model and two large neighborhood approaches are proposed to solve the CSPP in Section 3 and Section 4, respectively. In Section 5, we first explain the generation of problem instances and then analyze the parameter setting. After that, the results of the computational experiments are presented to evaluate the proposed approaches. Finally, concluding remarks and plans for further research are outlined in Section 7.

\section{Related works}

In recent years, research on cloud computing was focused, to a large extent, on the provider perspective (Heilig \& Voß, 2014b), increasingly resulting in innovative applications for managing and offering computing resources efficiently in terms of revenue, cost, and energy consumption (see, e.g., Bansal, Lee, Nagarajan, \& Zafer, 2015; Medernach \& Sanlaville, 2012; Püschel et al., 2015). Quite recently, cloud consumer-related problems have become increasingly important (Marston, Li, Bandyopadhyay, Zhang, \& Ghalsasi, 2011). In this section, we first review relevant works specifically related to optimization approaches for selecting and purchasing virtual machines in cloud environments from a consumer perspective. After analyzing the covered aspects in related problem descriptions, we compare the works using five main categories. 
Chaisiri, Lee, and Niyato (2009, 2012) present a stochastic programming approach to support the provisioning of virtual machines using both on-demand and reservation plans by taking into account demand and price uncertainties. Their model aims at reserving a cost-optimal number of resources in the first period and extend it, if necessary, by on-demand resources in subsequent periods. To obtain solutions, the authors consider different approaches including a deterministic equivalent formulation, sample-average approximation, and Benders decomposition. Van den Bossche, Vanmechelen, and Broeckhove (2010) specifically consider hybrid cloud architectures where tasks are outsourced from an internal data center (i.e., private cloud) to a public cloud provider in times of heavy loads. The authors present a binary integer programming model to support hybrid cloud scheduling. In Andrzejak, Kondo, and Yi (2010), a brokerage architecture for supporting the option of cloud resource bidding is addressed by proposing a binary integer programming model. A prediction model, taking into account the historical prices of a respective cloud provider, is used to predict the dynamic prices of virtual machines. Pandey, Wu, Guru, and Buyya (2010) propose a brokerage scheme for supporting application workflows consisting of orchestrated tasks that require a sequence-constrained scheduling. The authors represent the application workflow as directed acyclic graph and present a particle swarm optimization (PSO) approach to optimize the mapping of applications to cloud resources in a way that execution times and communication costs among applications are minimized. Lucas-Simarro, Moreno-Vozmediano, Montero, and Llorente (2011, 2013) present a cloud brokerage architecture for multi-cloud environments and consider a binary integer programming formulation. Besides common characteristics, the proposed scheduling algorithms consider reallocation or load balancing constraints as well as static and dynamic conditions, such as regarding resource prices. Those prices are estimated based on the average price and current price trend. The heterogeneous characteristics of cloud resources as well as dynamic demands are not considered. The work of Guo et al. (2012) addresses the problem of data placement in multiple clouds by specifically considering the communication costs between different clouds. The authors map the data of tasks to virtual computing resources and propose a PSO algorithm to minimize the processing and data transfer costs. Gutierrez-Garcia and Sim (2012) investigate a genetic algorithm for scheduling budgetand deadline-constrained application tasks. Moreover, the authors propose a multi-agent system to provision virtual machines and coordinate their use in a distributed manner. Tordsson, Montero, Moreno-Vozmediano, and Llorente (2012) formulate the problem of assigning and scheduling tasks to cloud resources as a variant of the generalized assignment problem. Similar to the approach of Lucas-Simarro et al. (2011, 2013), the authors consider load balancing constraints for multi-cloud environments, which maintain a certain number of virtual machines in each cloud. As in the problem addressed in Chaisiri et al. (2009, 2012), different scheduling heuristics are proposed in Shen, Deng, Iosup, and Epema (2013) to incorporate the option of on-demand and reservation plans. Coutinho, Drummond, and Frota (2013) propose the Cloud Resource Management Problem (CRMP), which is a multicriteria optimization model taking into account both cost and performance preferences of consumers. The authors present an integer programming formulation, which considers resource demands of applications, budget limits, and various virtual machine types. A Greedy Randomized Adaptive Search Procedure (GRASP) is proposed for solving the problem. In Coutinho, Drummond, Frota, and de Oliveira (2015), the authors extend the problem formulation by considering communication costs between application tasks deployed in different clouds. To facilitate real-time decision making and a higher solution quality, a Biased Random Key Genetic Algorithm (BRKGA) is proposed for solving the CRMP in
Heilig et al. (2016). This evolutionary approach noticeably outperforms recent approaches by providing new best-known solutions within small computation times in the millisecond range for all problem instances.

By extensively analyzing the respective problems and assumptions, we identify important aspects being covered in the relevant works and use them to compare the different approaches. As depicted in Table 1, we group the identified aspects into five main categories. The first three categories cover provider-specific aspects considered in the literature (supply side), including the configuration of the cloud environment, resource characteristics, and pricing schemes. The remaining two categories contain specific requirements and objectives from the perspective of consumers. This includes budget limits, resource demands, performance requirements, and specific application architectures. In the following, we briefly discuss how related works have addressed those different aspects.

- Cloud environment and brokerage: Several works consider a multi-cloud environment where a cloud brokerage scheme is used to mediate between the consumer and multiple providers by purchasing virtual machines according to the consumer's requirements. In the relevant works, brokers exclusively act on behalf of one consumer. Chaisiri et al. (2009, 2012) propose a cloud brokerage scheme to support the provisioning of virtual machines based on a reservation plan. Van den Bossche et al. (2010) specifically consider hybrid cloud architectures. Moreover, Coutinho et al. (2013, 2015) assume a limited number of virtual machines, which can be considered as a private cloud setting.

- Cloud resource characteristics: Cloud resource characteristics are expressed by physical properties, mainly in terms of computing power (e.g., number of cores, instructions per second), memory, and storage. Other important cloud resource characteristics, such as the operating system and deployment region, have not been taken into account. These characteristics can be selected in common public cloud environments and usually have a high impact on the price of the virtual machine. While most of the works consider related problems in a single-period fashion, a few works address dynamic aspects by considering varying resource demands and dynamic prices in multi-period scenarios (Andrzejak et al., 2010; Lucas-Simarro et al., 2011; 2013; Pandey et al., 2010; Tordsson et al., 2012).

- Cloud pricing: The cost of purchasing virtual machines is closely linked to the pricing model of the cloud provider. On-demand pricing, also referred to as pay-as-you-go pricing, is the most common pricing model in public clouds. The consumer pays a fixed price per time unit (e.g., hour) for using virtual machines. Pricing varies depending on the capacities and deployment region of the different virtual machine types. The majority of relevant works model on-demand pricing and use real prices of at least one provider (e.g., from Amazon EC2). However, the used data is rather restricted and it is not possible to fully cover and analyze the real portfolio of cloud providers. The impact of the deployment region on the price, for example, is currently not taken into account. Some works further consider extra bandwidth costs for transferring data to/from virtual machines (Van den Bossche et al., 2010; Coutinho et al., 2015; Guo et al., 2012; Heilig et al., 2016; Pandey et al., 2010). Andrzejak et al. (2010) take into account the option of bidding for virtual machines, referred to as spot instances, and support consumers in deciding on the bid prices considering cost and performance requirements. Reserving virtual machines is considered as another purchasing option. That is, the consumer gets a discount on the hourly price of virtual machines when reserving them upfront. In Chaisiri et al. (2009, 2012), the cloud 
Table 1

Cloud service selection aspects covered in the literature.

\begin{tabular}{|c|c|c|c|c|c|c|c|c|c|c|c|c|c|c|}
\hline & $\begin{array}{l}\text { Chaisiri et } \\
\text { al. (2009, } \\
\text { 2012) }\end{array}$ & $\begin{array}{l}\text { Van den } \\
\text { Bossche et } \\
\text { al. (2010) }\end{array}$ & $\begin{array}{l}\text { Andrzejak } \\
\text { et al. } \\
\text { (2010) }\end{array}$ & $\begin{array}{l}\text { Pandey et } \\
\text { al. (2010) }\end{array}$ & $\begin{array}{l}\text { Lucas- } \\
\text { Simarro et } \\
\text { al. (2011) }\end{array}$ & $\begin{array}{l}\text { Guo et al. } \\
\text { (2012) }\end{array}$ & $\begin{array}{l}\text { Gutierrez- } \\
\text { Garcia and } \\
\text { Sim (2012) }\end{array}$ & $\begin{array}{l}\text { Tordsson } \\
\text { et al. } \\
\text { (2012) }\end{array}$ & $\begin{array}{l}\text { Lucas- } \\
\text { Simarro et } \\
\text { al. (2013) }\end{array}$ & $\begin{array}{l}\text { Shen et al. } \\
\text { (2013) }\end{array}$ & $\begin{array}{l}\text { Coutinho } \\
\text { et al. } \\
\text { (2013) }\end{array}$ & $\begin{array}{l}\text { Coutinho } \\
\text { et al. } \\
\text { (2015) }\end{array}$ & $\begin{array}{l}\text { Heilig et } \\
\text { al. (2016) }\end{array}$ & $\begin{array}{l}\text { CSPP (this } \\
\text { work) }\end{array}$ \\
\hline \multicolumn{15}{|l|}{ Cloud Environment } \\
\hline Multi-cloud / cloud broker & - & - & & & - & - & & - & - & & & - & - & - \\
\hline Communication between clouds & & & & & & - & & & & & & & & \\
\hline Communication between virtual machines & & & & & & & & & & & & - & - & \\
\hline Reallocation possible & & & & & - & & & & - & & & & & \\
\hline Cloud load balancing & & & & & - & & & - & - & & & & & \\
\hline Resource bidding possible & & & - & & & & & & & & & & & \\
\hline \multicolumn{15}{|l|}{ Cloud Resource Characteristics } \\
\hline Computing power & - & - & - & - & & - & - & - & - & - & - & - & - & - \\
\hline Memory & & - & & & & & & - & - & & - & - & - & - \\
\hline Storage & - & & & - & & & & - & - & & - & - & - & - \\
\hline Operating System & & & & & & & & & & & & & & - \\
\hline Location & & & & & & & & & & & & & & - \\
\hline \multicolumn{15}{|l|}{ Cloud Pricing } \\
\hline On-demand pricing (static) & & - & & - & & - & - & - & & - & - & - & - & - \\
\hline Reserved pricing (static) & - & & & & & & & & & - & & & & \\
\hline Dynamic prices & - & & - & • & • & & & & - & & & & & \\
\hline Extra bandwidth costs & & - & & - & & - & & & & & & - & - & \\
\hline Real-world prices & & & $\bullet$ & & $\bullet$ & $\bullet$ & & $\bullet$ & $\bullet$ & $\bullet$ & $\bullet$ & $\bullet$ & $\bullet$ & $\bullet$ \\
\hline \multicolumn{15}{|l|}{ Consumer and Application Requirements } \\
\hline Consumer Budget & & & - & & & & - & - & - & & - & - & - & \\
\hline Compute power & - & & & & & - & - & & & - & - & - & - & - \\
\hline Memory & & & & & & & & & & & - & - & - & - \\
\hline Storage & • & & & & & & & & & & - & - & - & - \\
\hline Network bandwidth & - & - & & - & & - & & & & & & - & - & \\
\hline Operating system & & & & & & & & & & & & & & - \\
\hline Location & & & & & & & & & & & & & & - \\
\hline Arrival time & & & & & & & & & & - & & & & \\
\hline Execution deadline & & - & & & & & - & & & - & & & & - \\
\hline Execution time & & - & - & • & & - & & & - & & & & & \\
\hline Subtasks & & - & & • & & & & & & & & & & \\
\hline Task dependencies & & & & - & & & & & & & & & & \\
\hline $\begin{array}{l}\text { Dynamic resource demands } \\
\text { Objective Function }\end{array}$ & $\bullet$ & & & $\bullet$ & & & & & $\bullet$ & $\bullet$ & & & & \\
\hline Execution costs & - & - & - & - & - & - & - & - & - & - & - & - & - & - \\
\hline Execution time & & & & & & - & - & - & - & & - & - & - & \\
\hline
\end{tabular}


broker first generates a reservation plan with the option to purchase additional virtual machines if the number of reserved ones is exceeded in the utilization phase. The authors assume prices known a priori and dynamic demands, though they do not use real cloud data. The implications of long reservation periods (e.g., one year - which is a common reservation period) are not discussed. Shen et al. (2013) propose scheduling policies to efficiently utilize different types of already reserved virtual machines and additionally purchase on-demand ones.

- Consumer and application requirements / objective function: In most of the works, consumer and application requirements are represented in a static way. The required computing power is used as a main application requirement. Recent works further consider required memory and storage capacities as they have an essential impact on the overall deployment costs. Depending on the specific problem, some works further consider network bandwidth, dependencies between tasks and subtasks (Pandey et al., 2010), and dynamic resource demands (Chaisiri et al., 2009; 2012; Lucas-Simarro, Moreno-Vozmediano, Montero, \& Llorente, 2013; Pandey et al., 2010; Shen et al., 2013). Shen et al. (2013) further use different selection policies based on the arrival time of application tasks. Coutinho et al. (2013, 2015) aggregate the resource requirements of individual tasks and thus assume that they can be flexibly divided for being executed in a distributed way. Whereas all works consider cost optimization, some works further take into account the execution time in the objective function by treating the objectives in separate policies (Gutierrez-Garcia \& Sim, 2012; Lucas-Simarro et al., 2011; 2013; Tordsson et al., 2012) or in a multi-criteria optimization fashion using a simple weighted sum approach (Coutinho et al., 2013; Coutinho et al., 2015; Guo et al., 2012; Heilig et al., 2016).

As indicated in Table 1, our work contributes to this line of research by proposing a novel cloud brokerage mechanism for multicloud environments. Compared to the related works, our approach allows the assignment of a flexible number of heterogeneous application tasks, allows the sharing of virtual machines, and considers additional cloud resource characteristics and consumer requirements. We specifically consider the fact that virtual machine types are offered in various regions and locations for different prices. Taking into account the high importance of the deployment region, having a significant impact on data protection regulations and the place of jurisdiction (Subashini \& Kavitha, 2011), the cloud brokerage mechanism takes into account the region preference of consumers. The impact of the provisioned operating system on the price of a virtual machine is further taken into account. The paper also differentiates between different types of demand profiles in the generated problem instances, covering standard, memoryintensive, and compute-intensive application tasks. As presented in the following, we propose a mixed-integer programming model and two large neighborhood search approaches to facilitate an efficient assignment of application tasks to various virtual machine types of different cloud providers.

\section{Cloud service purchasing problem}

Nowadays, there are many cases where cloud consumers aim to execute application tasks with different resource requirements on virtual machines in the cloud. This type of assignment problem increasingly occurs in real-world applications and has become especially important in the era of cloud computing. Two prominent examples, intensively discussed in recent years and increasingly gaining the attention of the industry, involve the deployment of microservice architectures (see, e.g., Familiar, 2015) and big data processing (see, e.g., Chen, Mao, \& Liu, 2014; Heilig \& Voß, 2017),

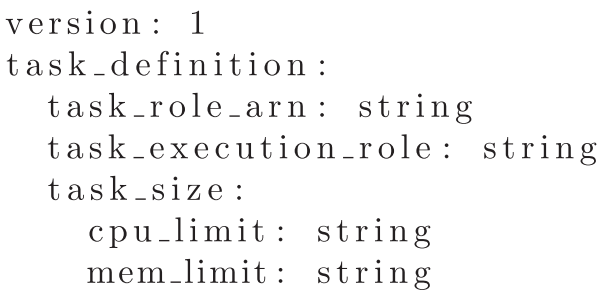

Listing 1. ECS task definition (AWS, 2019).

both aiming to achieve a high scalability through an execution of isolated tasks in a distributed computing environment using cloud resources. To provide an example of a real-world application, we refer to a previous work where we have assessed, in collaboration with a large cloud provider, practical requirements for an automated deployment of elastic cloud services focusing on the Adobe Shared Cloud (Heilig et al., 2015). In the Adobe Shared Cloud, which is a PaaS built for supporting several cloud solutions (e.g., Creative Cloud, Acrobat.com, and the Digital Publishing Suite), microservices with individual resource requirements need to be globally deployed on virtual machines of different public cloud providers (e.g., AWS, Microsoft Azure). In this context, application task examples, mostly executed in an isolated way, are the ones for creating renditions, generating color themes, or performing the transcoding of videos, images, etc.

In recent years, cloud providers have extended their IaaS portfolio to further abstract from the virtual machine level. Instead of deploying applications directly on purchased virtual machines (for example in Amazon EC2), the consumer only has to define the task requirements, deploys application tasks as microservice (e.g., as Docker container) into a container repository (e.g., Amazon $\mathrm{ECR}^{1}$ ), and the cloud provider takes care of the provisioning of underlying resources, referred to as serverless computing (for an extensive introduction see, e.g., Baldini et al., 2017). As an example, some input parameters for a task definition used for AWS $\mathrm{ECS}^{2}$ /Fargate, launched end of 2017, are shown in Listing 1. However, using those cloud services leads to a higher vendor lock-in and cannot be applied in multi-clouds. Moreover, the control is fully with the cloud providers, meaning that cost reductions are mostly leading to a benefit for cloud providers and are only partially passed to the consumer.

For addressing those challenges and for assigning individual application tasks to virtual machine types in multi-cloud environments, this work is aimed at formulating a generic approach, which can be also used for other types of applications (e.g., scientific applications as considered in Heilig et al., 2016). Therefore, in this section, we propose the Cloud Service Purchasing Problem (CSPP) and present a respective mathematical model to address this assignment problem while considering important assumptions and constraints.

In the CSPP, we aim to assign a set of application tasks $A$ to virtual machines from a set of different virtual machine types $V$ offered by the different cloud providers along a given time horizon $T$. Moreover, each application task $a \in A$ has its own requirements regarding the processing capacity $g_{a}$, hard disk capacity $d_{a}$, memory capacity $m_{a}$, operating system $o_{a}$, region $r_{a}$, and deadline $t_{a}$. The applications' requirements have to be fulfilled by the assigned virtual machine(s). Hence, each virtual machine of type $v \in V$ provides an amount of giga floating operations per second (GFlops), $G_{v}$, which represents the processing capacity of the virtual machine provided in each second along the purchased time units in

\footnotetext{
1 Amazon Elastic Container Registry, see https://aws.amazon.com/ecr/.

2 Amazon Elastic Container Service, see https://aws.amazon.com/ecs/.
} 
Table 2

\begin{tabular}{ll} 
Parameters of the CSPP. \\
\hline Parameters & Description \\
\hline$A$ & Set of $|A|$ application tasks \\
$V$ & Set of $|V|$ virtual machine types \\
$L$ & Set of available regions \\
$S$ & Set of available operating systems \\
$T$ & Set of time periods \\
$c_{v i}^{j}$ & Costs of virtual machine type $v$ operated with operating system $j \in S_{v}$ in region $i \in L$ \\
$G_{v}$ & Amount of GFlops provided by virtual machine type $v \in V$ \\
$M_{v}$ & Amount of memory provided by virtual machine type $v \in V$ \\
$D_{v}$ & Amount of storage provided by virtual machine type $v \in V$ \\
$L_{v}$ & Set of available regions of virtual machine type $v \in V$ \\
$S_{v}$ & Set of available operating systems of virtual machine type $v \in V$ \\
$O_{v}^{i}$ & 1 if virtual machine type $v$ can be operated with operating system $i \in S_{v}, 0$ otherwise \\
$R_{v}^{j}$ & 1 if virtual machine type $v$ is available at region $j \in L_{v}, 0$ otherwise \\
$g_{a}$ & Amount of GFlop capacity required by application task $a \in A$ \\
$m_{a}$ & Amount of memory capacity required by application task $a \in A$ \\
$d_{a}$ & Amount of storage capacity required by application task $a \in A$ \\
$o_{a}^{j}$ & 1 if the application task must be executed in operating system $j \in S, 0$ otherwise \\
$r_{a}^{i}$ & 1 if the application task must be executed in region $i \in L, 0$ otherwise \\
$t_{a}$ & Deadline of application task $a$ \\
\hline
\end{tabular}

Table 3

Decision variables of the CSPP.

\begin{tabular}{ll}
\hline Decision variables & Description \\
\hline$x_{a v j}^{i t}$ & Application task $a \in A$ is executed on a virtual machine of type $v$ under operating system $j$ in region $i$ during time period $t$ \\
$w_{a a^{\prime} v}^{j i t}$ & Application tasks $a, a^{\prime} \in A$ are executed in the same virtual machine of type $v$ under operating system $j$ in region $i$ during time period $t$ \\
$z_{a v}^{j i}$ & Application task $a \in A$ is executed on a virtual machine of type $v$ under operating system $j$ in region $i$ \\
$y_{v j}^{i}$ & Number of virtual machines of type $v \in V$ purchased under operating system $j \in S_{v}$ in region $i \in L_{v}$ \\
$\delta_{a v j}^{i t}$ & Application task $a \in A$ shares with other tasks its assigned virtual machine of type $v$ at time period $t$ under operating system $j$ in region $i$ \\
\hline
\end{tabular}

hours. Further, the virtual machine is provisioned with memory $M_{v}$ and storage capacity $D_{v}$. Also, each virtual machine must be provisioned with a specific operating system $O_{v}$ (e.g., Windows, Linux) and needs to be deployed in a specific region $R_{v}$ (e.g., Europe, US, Asia). The objective of this problem is to minimize the total costs for purchasing virtual machines in order to execute application tasks issued by the cloud consumers. All input parameters and decision variables used in the model are depicted in Tables 2 and 3 , respectively. In the following we provide the assumptions specified for this problem:

- Each application task $a \in A$ can be assigned to one type of virtual machine $v \in V$.

- The memory requirements $m_{v}$ of each application task $a$ have to be fulfilled by the assigned virtual machine $v \in V$ at each time period $t \in T$ to avoid paging.

- The disk requirements $d_{v}$ of each application task $a$ have to be fulfilled by the assigned virtual machine $v \in V$ at each time period $t \in T$. If more than one application task is executing over the same virtual machine, the sum of the disk capacity requirements can be at most the capacity provided by the virtual machine, $D_{v}$, to avoid data transfers in-between the executions of those application tasks.

- The total processing amount $g_{a}$ required by each application task $a \in A$ has to be provided by its assigned virtual machine $v \in V$ before its deadline. In that regard, if more time periods are required to process a given application task, the provision of GFlops has to be performed within the given scheduled period.

- The execution of each $a \in A$ on its assigned virtual machine $v \in$ $V$ has to meet the region and operating system characteristics requested by the application task.

- Each virtual machine type $v \in V$ can be provisioned in any quantity at any time.
- The performance of computing resources is assumed to be constant over time.

- As it has been done in several related works (see Section 2), we model the application as isolated task without dependencies to other tasks.

- As currently common in cloud environments, resource capacities (e.g., CPU, memory) of virtual machine types are fixed, but the purchased number can be considered unlimited, especially in multi-cloud environments, where each cloud provider operates a huge number of datacentres around the globe.

- The application task requirements are known a priori.

Furthermore, to better illustrate this problem, an example of a solution for the CSPP is shown in Fig. 1. In this example, on the left side, we report three applications, i.e., $A=\left\{a_{1}, a_{2}, a_{3}\right\}$, where inside each box, we report the corresponding processing $(g)$, memory $(m)$, and disk $(d)$ requirements. On the other hand, to assign applications to virtual machines, we have two types of virtual machines $V=\left\{v_{1}, v_{2}\right\}$ for which we depict the corresponding computational processing $(G)$, memory $(M)$, and disk $(D)$ capacity as well as the corresponding price per time unit $(c)$. For this example, we assume that the region and operating system requirements are already satisfied by the set of provided virtual machines. The red arrows indicate the assignment without the sharing option, while the blue arrows indicate the same scenario when sharing is allowed. As can be checked in the example, four virtual machines are used when sharing is not allowed, i.e., two virtual machines of type $v_{1}$ and two of type $v_{2}$. In the example with sharing, three virtual machines are required, i.e., one virtual machine of type $v_{1}$ and two of type $v_{2}$. In the case without sharing, the second instance of $v_{2}$ is underutilized, i.e., 2/20; when sharing is considered, on the other hand, its utilization increases to 10/20. Finally, the objective function value of the solution without sharing is 50 , while it is decreased to 40 when sharing is allowed. 


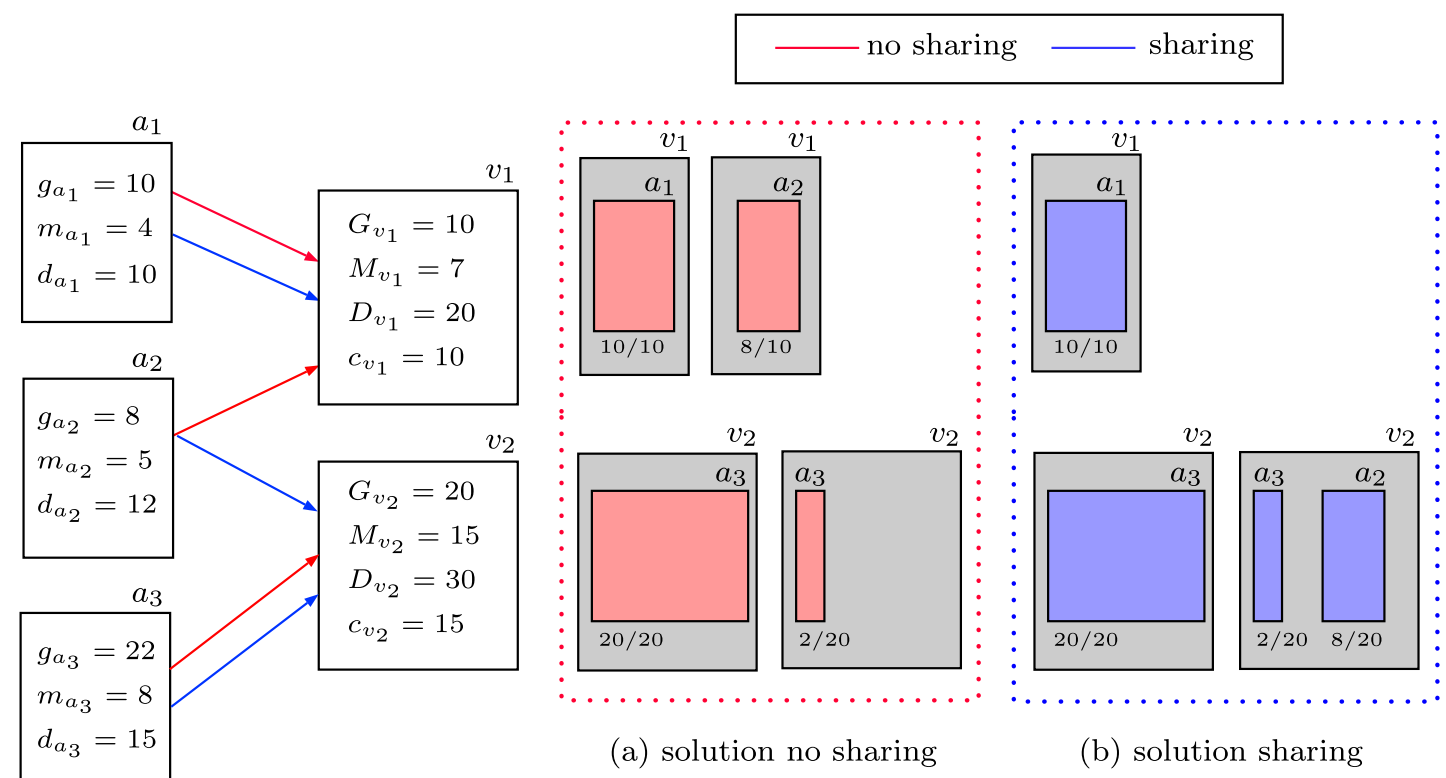

Fig. 1. Solution example with and without sharing for the CSPP with 3 applications and 2 virtual machines.

The mathematical formulation of the CSPP is stated as follows.

(CSPP) minimize $\sum_{v \in V} \sum_{j \in S_{v}} \sum_{i \in L_{v}} c_{v i}^{j} y_{v j}^{i}$

subject to the following constraints:

$\sum_{v \in V} \sum_{j \in S_{v}} \sum_{i \in L_{v}} z_{a v}^{j i}=1 \quad \forall a \in A$

$\sum_{v \in V} \sum_{j \in S_{v}} \sum_{i \in L_{v}} x_{a v j}^{i t} \leq 1 \quad \forall a \in A, \forall t \in T$

$\sum_{t \in T} x_{a v j}^{i t} \leq H V \cdot z_{a v}^{j i} \quad \forall a \in A, \forall v \in V, \forall j \in S_{v}, \forall i \in L_{v}$

$\sum_{t \in T} x_{a v j}^{i t} \geq z_{a v}^{j i} \quad \forall a \in A, \forall v \in V, \forall j \in S_{v}, \forall i \in L_{v}$

$\sum_{v \in V} \sum_{j \in S_{v}} \sum_{i \in L_{v}} z_{a v}^{j i}\left(M_{v}-m_{a}\right) \geq 0 \quad \forall a \in A$

$\sum_{v \in V} \sum_{a^{\prime} \in A, a \neq a^{\prime}} \sum_{j \in S_{v}} \sum_{i \in L_{v}} w_{a a^{\prime} v}^{j i t} d_{a^{\prime}} \leq \sum_{v \in V} \sum_{j \in S_{v}} \sum_{i \in L_{v}}\left(D_{v}-d_{a}\right) x_{a v j}^{i t} \quad \forall a \in A, \forall t \in T$

$2 \cdot w_{a a^{\prime} v}^{j i t} \leq x_{a v j}^{i t}+x_{a^{\prime} v j}^{i t} \quad \forall a, a^{\prime} \in A, a \neq a^{\prime}, \forall t \in T, \forall v \in V, \forall j \in S_{v}$,

$\forall i \in L_{v}$

$w_{a a^{\prime} v}^{j i t}=w_{a^{\prime} a v}^{j i t} \quad \forall a, a^{\prime} \in A, a \neq a^{\prime}, \forall t \in T, \forall v \in V, \forall j \in S_{v}, \forall i \in L_{v}$

$w_{a a^{\prime} v}^{j i t}+w_{a a^{\prime \prime} v}^{j i t}-w_{a^{\prime} a^{\prime \prime} v}^{j i t} \leq 1 \quad \forall a, a^{\prime}, a^{\prime \prime} \in A, a \neq a^{\prime} \neq a^{\prime \prime}, \forall t \in T$,

$\forall v \in V, \forall j \in S_{v}, \forall i \in L_{v}$

$\sum z_{a v}^{j i} g_{a} \leq G_{v} y_{v j}^{i} \quad \forall v \in V, \forall j \in S_{v}, \forall i \in L_{v}$

$G_{v}^{a \in A} \sum_{a \in A} \sum_{t \in T}\left(x_{a v j}^{i t}-\delta_{a v j}^{i t}\right) \geq \sum_{a \in A} z_{a v}^{j i} g_{a} \quad \forall v \in V, \forall j \in S_{v}, \forall i \in L_{v}$

$\delta_{a v j}^{i t} \geq w_{a a^{\prime} v}^{j i t} \quad \forall a, a^{\prime} \in A, a<a^{\prime}, \forall v \in V, \forall t \in T, \forall j \in S_{v}, \forall i \in L_{v}$

$$
\begin{aligned}
& \delta_{a v j}^{i t} \leq \sum_{a^{\prime}=1, a<a^{\prime}}^{A} w_{a a^{\prime} v}^{j i t} \quad \forall a \in A, \forall v \in V, \forall t \in T, \forall j \in S_{v}, \forall i \in L_{v} \\
& \sum_{a \in A} \sum_{t \in T}\left(\sum_{j \in S_{v}} \sum_{i \in L_{v}} x_{a v j}^{i t}-\delta_{a v j}^{i t}\right) \leq \sum_{j \in S_{v}} \sum_{i \in L_{v}} y_{v j}^{i} \quad \forall v \in V \\
& \sum_{v \in V} \sum_{j \in S} \sum_{i \in L}\left(O_{v}^{j}-o_{a}^{j}\right) z_{a v}^{j i}=0 \quad \forall a \in A \\
& \sum_{v \in V} \sum_{j \in S} \sum_{i \in L}\left(R_{v}^{i}-r_{a}^{i}\right) z_{a v}^{j i}=0 \quad \forall a \in A \\
& t \cdot \sum_{j \in S_{v}} \sum_{i \in L_{v}} x_{a v j}^{i t} \leq t_{a} \quad \forall a \in A, \forall t \in T, \forall v \in V \\
& x_{a v j}^{i t} \in\{0,1\} \quad \forall a \in A, \forall v \in V, \forall j \in S_{v}, \forall i \in L_{v}, \forall t \in T \\
& w_{a a^{\prime} v}^{j i t} \in\{0,1\} \quad \forall a, a^{\prime} \in A, \forall v \in V, \forall j \in S_{v}, \forall i \in L_{v}, \forall t \in T \\
& z_{a v}^{j i} \in\{0,1\} \quad \forall a \in A, \forall v \in V, \forall j \in S_{v}, \forall i \in L_{v} \\
& y_{v j}^{i} \in \mathbb{Z}_{0}^{+} \quad \forall v \in V, \forall j \in S_{v}, \forall i \in L_{v} \\
& \delta_{a v j}^{i t} \in\{0,1\} \quad \forall a \in A, \forall v \in V, \forall t \in T
\end{aligned}
$$

The objective function of the CSPP model (1) seeks to minimize the costs for purchasing virtual machines. While constraints (2) establish that each application task has to be assigned to only one virtual machine type, constraints (3) set that each application task can be performed in at most one virtual machine at each time period. Hence, the same application task cannot be run in parallel. Constraints (4) and (5) link variables $z_{a v}^{j i}$ and $x_{a v j}^{i t}$, where $H V$ is a high-enough value. Thus, by means of these constraints we can isolate variable $x$ from the time dimension by means of having variable $z$. Constraints (6) and (7) state that the minimum task resource demands in terms of memory and storage capacity have to be satisfied at each time period for each application task. As can be observed, constraints (6) imply that each application task cannot surpass the memory capacity provided by the virtual machine and constraints (7) ensure the same for the disk capacity 
considering all the application tasks executed in that virtual machine. Constraints (8)-(10) establish the option of sharing a virtual machine if the application tasks are executed together during time period $t$ by means of variables $w_{a a^{\prime} v^{j}}^{j i t}$ Namely, if two applications are assigned to the same type of virtual machine, then both can share it $w_{a a^{\prime} v}^{j i t}$ as shown in constraints (8). Constraints (9) establish the symmetry of the value of variable $w_{a a^{\prime} v}^{j i t}$ when two applications share the same virtual machine. Constraints (10) set the value of $w_{a a^{\prime} v}^{j i t}$ when more than two applications share the same virtual machine. Constraints (11) ensure that the total amount of GFlops required by all application tasks assigned to a virtual machine has to be completely satisfied. Constraints (12) establish that the amount of GFlops provided by the virtual machines satisfies the assigned applications along the time and ensures that the share is sufficient for satisfying their requirements. Constraints (13)-(14) set the auxiliary variables $\delta_{a v j}^{i t}$ which indicate whether an application task is sharing a given virtual machine with other tasks at a given time period. As can be checked in the constraints, when an application task is sharing a given virtual machine, for the application task with the higher identifier $\delta_{a v j}^{i t}$ equals zero in order to appropriately consider the purchase of the virtual machine in constraints (15). Constraints (15) define the lower bound on variable $y_{v j}^{i}$, i.e., the total number of virtual machines of type $v$ used, considering their assignment $\left(x_{a v j}^{i t}\right)$ and share $\left(\delta_{a p j}^{i t}\right)$ along the time. Constraints (16) determine that the required operating system is used. Similarly, constraints (17) set that each application has to be executed in the required region. Constraints (18) state that each application task $a \in A$ has to be executed before its given deadline $t_{a}$. Finally, constraints (19)-(23) define the decision variable types. Moreover, it should be noted that the option of sharing can be disabled, as depicted in the example of Fig. 1 (i.e., red arrows), by fixing the variables $w_{a a^{\prime} v}^{j i t}$ to zero.

\section{Adaptive large neighborhood search for the CSPP}

In this section, we present a metaheuristic framework using large neighborhood approaches and explain how they are applied for solving the CSPP. Adaptive Large Neighborhood Search (ALNS), proposed in Ropke and Pisinger (2006), is a constructive metaheuristic that extends the idea of Large Neighborhood Search (LNS) proposed by Shaw (1998). In comparison with local search, which makes small changes to current solutions when exploring the solution space, LNS modifies large parts of an incumbent solution by consecutively applying a destroy and repair heuristic in each iteration. The idea is similar to the ruin and recreate principle proposed in Schrimpf, Schneider, Stamm-Wilbrandt, and Dueck (2000). It has been demonstrated that using large neighborhood moves instead of smaller ones is particularly suited for more complex optimization problems with many constraints (see, e.g., Ropke \& Pisinger, 2006; Schrimpf et al., 2000), as it is the case for the CSPP.

ALNS builds upon the concept of LNS by allowing to select the destroy and repair heuristic from a pool of several available heuristics in a probabilistic and adaptive fashion taking into account their previous performance. Instead of using more complicated methods (e.g., branch-and-bound), simple and fast heuristics are preferred. The acceptance and stopping criteria of the ALNS are obtained by embedding the metaheuristic into a simulated annealing framework (Ritzinger \& Puchinger, 2013). LNS, in contrast, uses a simple descent approach (Ropke \& Pisinger, 2006). ALNS has been successfully applied to assignment problems (see, e.g., Brandt, Speck, \& Völker, 2016; de León, Lalla-Ruiz, Melián-Batista, \& Moreno-Vega, 2017; Mauri, Ribeiro, Lorena, \& Laporte, 2016; Yu, Zhang, \& Lau, 2017). In the following, we briefly explain the metaheuristic framework and its relevant components, including the problem-dependent heuristics for designing the ALNS for the CSPP.

\subsection{Generic search process}

To better understand the metaheuristic approaches, we briefly explain the main components and steps during the search process of LNS and ALNS. Fig. 2 depicts the search process for both metaheuristics. The components of the ALNS, extending the LNS, are embodied in dotted line boxes. As LNS is contained in ALNS, we explain the notations and general search process of the ALNS metaheuristic and emphasize the differences to LNS. The specific components (1)-(3) are explained in subsequent subsections. The individual constructive, destroy, and repair heuristics, used to instantiate the framework, are explained in Section 4.4. Note that the pseudocode of the used metaheuristic is provided in Appendix A.

The search process starts with generating an initial solution $S$ by means of a constructive heuristic (see Section 4.2). At the be-

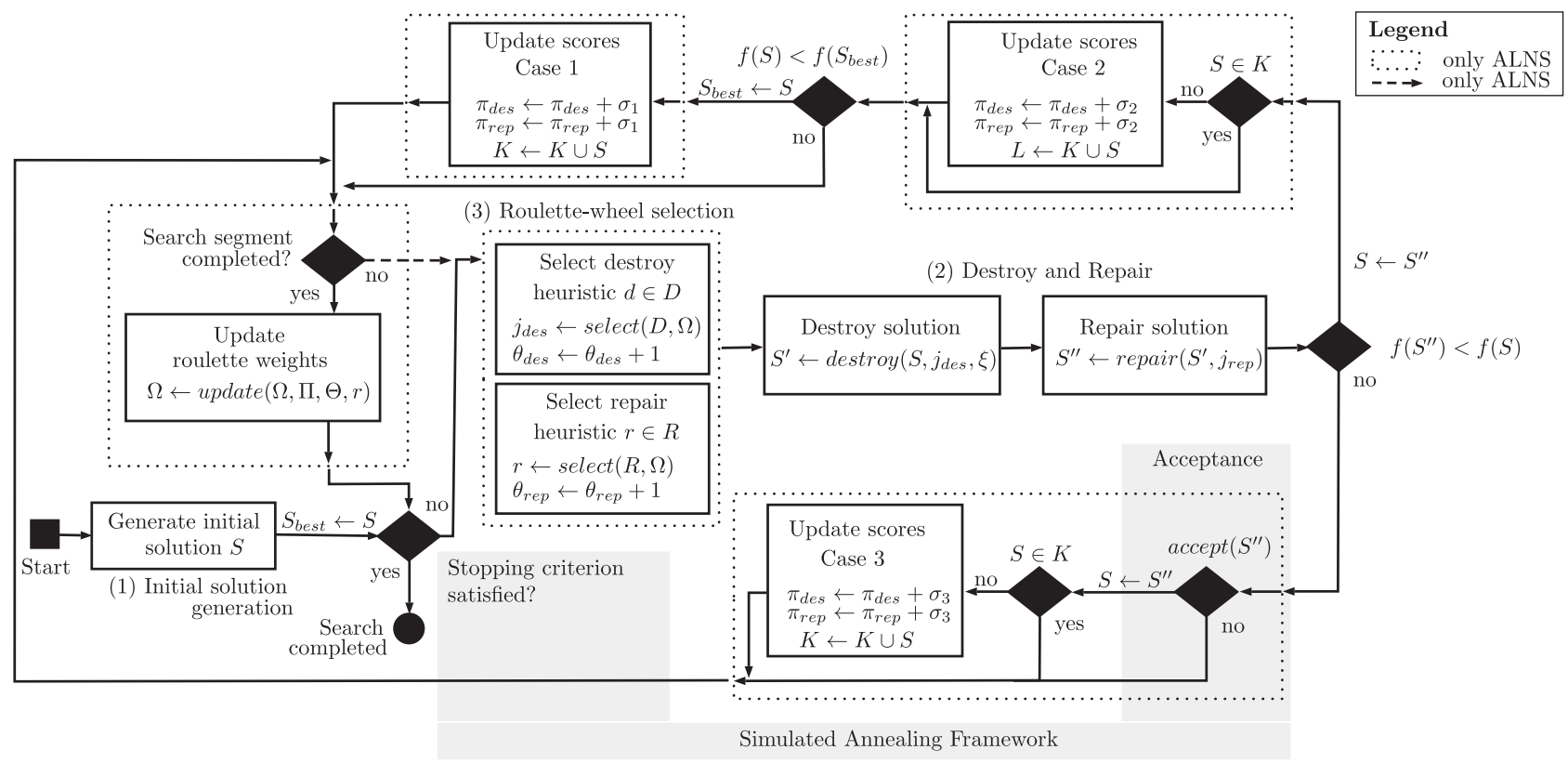

Fig. 2. Metaheuristic framework of ALNS and LNS. 
ginning, the best known solution is set to the initial one $\left(S_{\text {best }} \leftarrow S\right)$. To modify a current solution $S$, a destroy heuristic $j_{\text {des }}$ and repair heuristic $j_{\text {rep }}$ need to be selected. Whereas $j_{\text {des }}$ and $j_{\text {rep }}$ are predefined in LNS, the roulette-wheel selection principle (see Section 4.5) is used in ALNS to select one out of a set of destroy heuristics $D$ and one out of a set of repair heuristics $R$, such that $j_{\text {des }} \in D$ and $j_{\text {rep }} \in R$. For updating the roulette weights in a later step, the number of times the selected destroy and repair heuristics have been used, denoted by $\theta_{d}$ and $\theta_{r}$, respectively, is furthermore updated. First, the selected destroy heuristic $j_{\text {des }}$ is applied to the current solution $S$ to remove a number of assignments $\xi$ (in \%) from the solution. The resulting solution $S^{\prime}$ is repaired to a feasible solution $S^{\prime \prime}$ by applying the defined repair heuristic $j_{\text {rep }}$. Note that the combination of destroy and repair heuristic defines the neighborhood (see Section 4.3). If the objective function value $f\left(S^{\prime \prime}\right)$ is better than $f(S)$, the current solution is updated to the new solution $\left(S \leftarrow S^{\prime \prime}\right)$. If it is also better than the best known solution $S_{\text {best }}$, the best known solution is updated to the new current solution $\left(S_{\text {best }} \leftarrow S\right)$. For having the possibility to also accept worse solutions, avoiding being trapped in a local minimum, an acceptance function accept $(\cdot)$ is applied. Dependent on the following cases, the performance scores $\pi_{\text {des }}$ and $\pi_{\text {rep }}$ of the heuristics $j_{\text {des }}$ and $j_{\text {rep }}$, respectively, need to be updated in each iteration.

- Case 1: A new best solution $S_{\text {best }}$ has been found. The scores $\pi_{\text {des }}$ and $\pi_{\text {rep }}$ of the involved heuristics are increased by $\sigma_{1}$, which is typically the highest score increment.

- Case 2: A new solution improving the current solution $S$ has been found. The respective scores are increased by $\sigma_{2}$, if the solution has not been found before $(S \notin K)$. If so, the set $K$, storing new and accepted solutions, is updated $(K \leftarrow K \cup S)$.

- Case 3: A worse solution has been accepted. The respective scores are increased by $\sigma_{3}$, if the solution has not been found before $(S \notin K)$. As in the previous case, $K$ is updated.

The updated counter and score for each heuristic $j$ is used to measure its individual performance during a fixed number of iterations, denoted as search segment. After a search segment, the score vector $\Pi=\left[\pi_{1}, \pi_{2}, \ldots, \pi_{j}, \ldots, \pi_{|D+R|}\right]$ and counter vector $\Theta=\left[\theta_{1}, \theta_{2}, \ldots, \theta_{j}, \ldots, \theta_{|D+R|}\right]$ together with a reaction factor $r$ are used to update the roulette weight vector $\Omega=$ $\left[\omega_{1}, \omega_{2}, \ldots, \omega_{j}, \ldots, \omega_{|D+R|}\right]$, which determines the probability of selecting heuristics in subsequent iterations (see Section 4.5). As depicted by a dashed line, indicating that this applies only to the ALNS, the number of iterations of a search segment need to be completed before the stopping criterion is checked. The stopping criterion determines when the search process is completed.

As previously mentioned, ALNS is embedded into a simulated annealing framework to define the acceptance and stopping criteria, as it has been successfully done in other ALNS approaches (Pisinger \& Ropke, 2007; 2010). Simulated annealing is a popular metaheuristic that extends local search through allowing movements leading to worse solutions in terms of objective function value. As a part of the acceptance function accept $\left(S^{\prime \prime}, S\right)$, the probability of accepting those solutions is computed as $e^{\left(-\frac{\Delta}{\text { Temp }}\right)}$, where $\Delta$ denotes the deterioration of the objective function value from solution $S$ to $S^{\prime \prime}$ and Temp is used as a control parameter. Initiated with a starting temperature, the temperature Temp is decreased by a cooling rate $\beta$ in each iteration (Temp $\leftarrow$ Temp $\beta \beta$ ) until it reaches a minimum temperature $T e m p_{\min }$. This condition $\left(T e m p_{\min } \geq T e m p\right)$ is used as a stopping criterion. In sum, ALNS applies three additional principles compared to a traditional local search: large neighborhood moves to explore large parts of the solution space, acceptance of worse solutions to avoid being trapped in a local minimum, and an adaptive use of heuristics (i.e., operators) to incorporate knowledge about the problem-dependent performance during the search process. Moreover, the adaptive selec- tion can lead to a more robust behavior over various types of problem instances.

\subsection{Initial solution generation: Greedy heuristic}

For generating an initial solution, indicated as component (1) in Fig. 2, as well as for comparing the quality of our proposed approach with a traditional programming approach, we use a greedy insertion heuristic that iteratively assigns the cheapest possible virtual machines to each application task by considering all requirements described in Section 3. The pseudocode of this procedure is depicted in Algorithm 1. As the solution $S$ is empty in the begin-

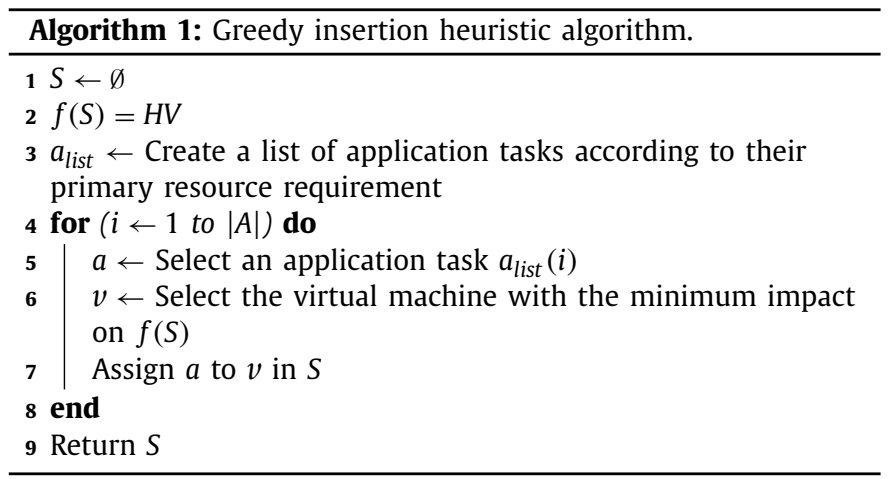

ning (line 1), a high-enough value $H V$ is initially associated with the solution $S$ (line 2). In line 3 , the list of application tasks, $a_{\text {list }}$, ordered according to their primary resource demand (e.g., GFlop) in descending order, is determined. That list is traversed in order to assign each application task $a$ to the virtual machine yielding the minimum increase of costs in the objective function value $f(S)$ (lines 4-8).

It may happen that the procedure for assigning an application task to a virtual machine finds several virtual machines with the same minimum impact on the objective function value $f(S)$. In this case, the virtual machine type with the best ratio in terms of GFlops, memory, and storage capacity should be selected. Therefore, when iteratively traversing through the list of available virtual machine types, a virtual machine type $v_{1}$ assigned to the current application task $a$ is replaced by virtual machine type $v_{2}$, yielding the same minimum impact on the objective function value, if (24) holds true.

$\frac{G_{v_{1}}-G_{v_{2}}}{\max \left(G_{v_{1}}, G_{v_{2}}\right)}+\frac{M_{v_{1}}-M_{v_{2}}}{\max \left(M_{v_{1}}, M_{v_{2}}\right)}+\frac{D_{v_{1}}-D_{v_{2}}}{\max \left(D_{v_{1}}, D_{v_{2}}\right)} \leq 0$

Note that the solution provided by this algorithm assigns one virtual machine to one application task; that is, no sharing of virtual machines is allowed. Nevertheless, this algorithm is used as a repair heuristic including the sharing option (see Section 4.4.4).

\subsection{Neighborhood}

The neighborhood of a solution is used for constructing a new solution in component (2) of the metaheuristic framework (see Fig. 2). In LNS and ALNS, the neighborhood of a solution $s$ is defined as the set of solutions that can be reached by first applying the selected destroy heuristic and then the selected repair heuristic. Thus, the definition of the neighborhood structure $\mathcal{N}$, assigning to every solution $s$ in the search space a set of neighbors $\mathcal{N}(s)$, is intrinsically determined by the combination of destroy and repair heuristics. This combination defines the changes that must be applied to a solution $s$ in order to generate all its neighbors. As multiple combinations are possible in ALNS, different neighborhood 
structures are used during the search in an adaptive fashion. While the applied destroy heuristic determines the open assignments of the solution and thus restricts the number of potential neighborhood solutions, the repair heuristic defines all possible neighborhood solutions by describing the process of assigning open application task requests to available or new virtual machines.

The size of the neighborhood is determined by the parameter $\xi$ with $0 \leq \xi \leq 1$. If $\xi$ is equal to zero, no search will be performed as no assignment is removed from the current solution. If $\xi$ is equal to one, on the other hand, the problem is resolved from scratch in each iteration as the solution is completely destroyed by removing all assignments. While a large $\xi$ might help to better explore the search space, a too large $\xi$ can lead to slow computational times and a lower solution quality in case a heuristic is used for the insertions (Ropke \& Pisinger, 2006). Therefore, it is important to separately analyze the impact of this parameter on the algorithmic performance (see Section 5.2).

\subsection{Destroy and repair heuristics}

To instantiate the metaheuristic framework for solving the CSPP, we have implemented several problem-specific destroy and repair heuristics. In the metaheuristic framework (see Fig. 2), those destroy and repair heuristics are related to the $\operatorname{destroy}(\cdot)$ and repair ( $)$ method of component (2), respectively.

\subsubsection{Shaw removal}

The first implemented destroy heuristic has been proposed in Shaw (1998). Here, we adapt this heuristic to suit the CSPP. The basic idea is to remove assignments using a relatedness measure $R\left(a_{1}, a_{2}\right)(25)$ that measures the similarity of two application tasks $a_{1}$ and $a_{2}$. A very small value of $R$ denotes a high relatedness between application tasks.

$R\left(a_{1}, a_{2}\right)= \begin{cases}\frac{\left|c_{a_{1}}-c_{a_{2}}\right|}{\max \left(c_{a_{1}}, c_{a_{2}}\right)}+\frac{\left|t_{a_{1}}-t_{a_{2}}\right|}{\max \left(t_{a_{1}}, t_{a_{2}}\right)}, & \text { if } \operatorname{reg}_{a_{1}}^{i}=\operatorname{reg}_{a_{2}}^{i} \wedge o s_{a_{1}}^{i}=o s_{a_{2}}^{i} \\ H V & \text { else }\end{cases}$

where $H V$ is a high-enough value. This value is used when the required region, reg, or operating system, os, of respective application tasks $a_{1}$ and $a_{2}$ differ. In all other cases, two main terms describe the relatedness of tasks. The first term compares the main capacity demands of two tasks. The compared capacities $c$ depend on the primary capacity requirement, which can be set to GFlop, memory, or storage. As the sole use of the GFlop criterion tends to provide the best performance over all problem instances, we only use this criterion to measure relatedness. The second term measures the temporal connectedness between two application tasks with respect to their execution deadlines $t_{a_{1}}$ and $t_{a_{2}}$, respectively.

The pseudocode for removing similar tasks using the relatedness measure is depicted in Algorithm 2. Line 4 indicates that the number of assignments to be removed is a non-negative value $|D|$. Parameter $p$ with $p \geq 1$ is used to control the randomness of selecting assignments to be removed. First, the heuristic randomly picks one assignment $r$ from a given solution $S$ (line 2 ) and adds it to the set $D$, initialized in line 3 to contain all assignments to be removed. Starting with the currently chosen assignment $r$, all remaining assignments are added to an array $L$ and sorted in ascending order according to their relatedness to $r$ (line 8) where the first element represents the one with the highest relatedness. A random number $y$ between zero and one is used to select one assignment from $L$ (line 9), which is added to $D$. By increasing the value of the parameter $p$, the likelihood of selecting dissimilar assignments can be reduced. Note that the parameter $p$ is used to control the randomness of selecting assignments to be removed. Since an adjustment, i.e., decrease, of the parameter $p$ does not tend to improve

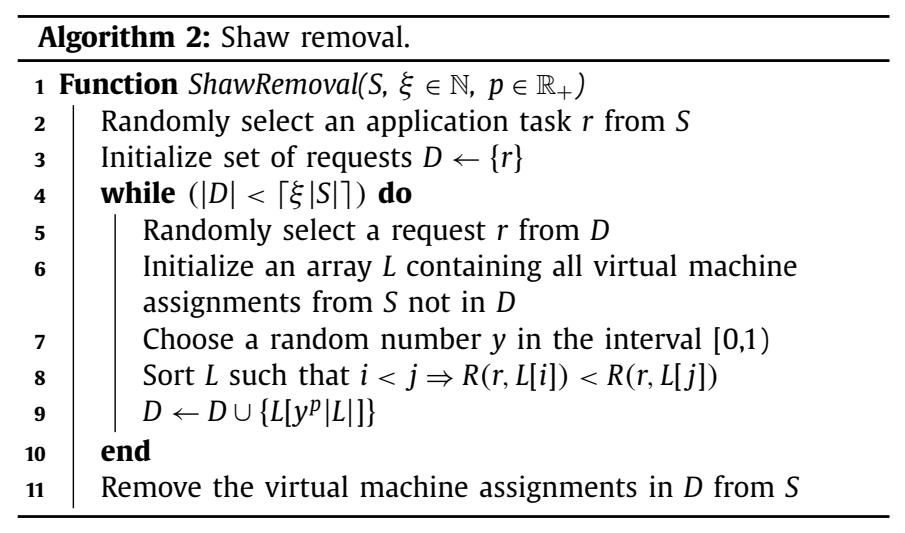

the performance of the algorithm for the problem at hand, we fix this parameter. The procedure is repeated until $\lceil\xi|S|\rceil$ assignments have been selected for being removed. For the sort and select procedures (line 8 and 9), we implement a quickselect algorithm to perform them, on average, in linear time.

\subsubsection{Worst removal}

Worst removal aims to remove an assignment that leads to high additional costs. In this respect, we define the cost of the application task as $\operatorname{cost}(a, s)=f(s)-f_{-a}(s)$ where $f_{-a}(s)$ denotes the cost of the solution without application task $a$. The cost might not be changed by removing a task in some cases where the task shares a virtual machine with other tasks as cloud providers charge for virtual machines on an hourly basis. In other words, the cloud provider still charges for freed-up rest capacity of the prior shared virtual machine.

The heuristic reuses some ideas from the Shaw destroy heuristic, such as regarding the selection of the assignment to be removed by introducing randomness through the parameter $p$ with $p \geq 1$. Algorithm 3 shows the procedure in pseudocode. Notice that

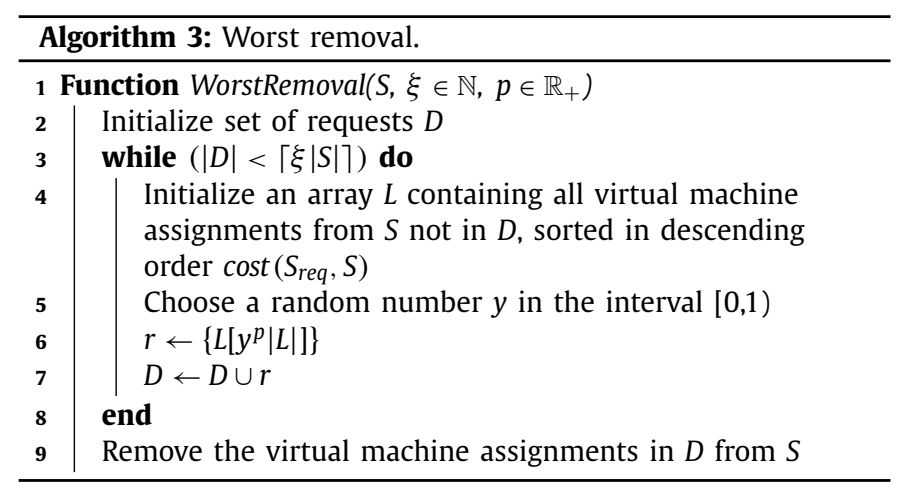

the sorting of array $L$ at each iteration (line 4 ) is avoided in the actual implementation of our algorithm by sorting only once and adding only application tasks that are not yet in $D$.

\subsubsection{Random removal}

Random removal randomly selects $\xi$ percent of the assignments for being removed from the solution. Therefore, it is a special case of the Shaw removal heuristic when $p=1$ (Pisinger \& Ropke, 2010).

\subsubsection{Greedy repair heuristic}

For defining a repair heuristic, we extend the greedy insertion heuristic presented in Section 4.2 by allowing the sharing of virtual machines among tasks. After a virtual machine has been assigned 
to a task, the rest capacity of that virtual machine in terms of GFlop is updated. The rest capacity is calculated as the difference between the overall amount of purchased GFlop capacity (booked virtual machine hours . GFlop/hour) and the assigned GFlop capacity.

In the subsequent iterations, it is further checked if the current task can be assigned to one of the already assigned virtual machines offering rest capacities. Therefore, we calculate the difference of required GFlop and available rest capacity and, in case the required capacity exceeds the available rest capacity of the virtual machine, determine the costs by calculating the additionally required execution hours. If several assigned virtual machines provide rest capacity, the best option in terms of costs is chosen and compared to the increase of the objective function value when using a dedicated virtual machine. Finally, the heuristic chooses the assignment with the lowest increase of the objective function value.

\subsection{Selection of destroy and repair heuristics}

The selection of destroy and repair heuristics follows the roulette-wheel selection principle, depicted as component (3) in Fig. 2. To determine the probability of selecting a specific destroy or repair heuristic, first, roulette weights $\omega_{j}$ need to be assigned to each heuristic $j$ as described above. Let $|H|$ be the number of considered heuristics, then a heuristic $j \in D \cup R$ is selected with probability

$$
\frac{\omega_{j}}{\sum_{i=1}^{|H|} \omega_{i}}
$$

Initially all roulette weights are set to one so that all heuristics receive the same chance of being selected. To calculate new roulette weights after performing a search segment (i.e., fixed number of iterations), an adaptive weight adjustment proposed in Pisinger and Ropke (2010) is used. The basic idea is to keep track of the performance of used heuristics during the search by introducing a score for the different heuristics. As explained in Section 4.1, the score for each selected heuristic can be increased by the score values $\sigma_{1}, \sigma_{2}, \sigma_{3}$ in each iteration depending on the respective case.

At the end of a search segment $s$, the roulette weights for all heuristics $j$ are updated using the recorded scores. Let $w_{j, s}$ be the roulette weight of heuristic $j$ used in segment $s$ calculated using formula (27). After weighing all heuristics $j$ equally in the first segment, new roulette weights for segment $s+1$ are calculated as follows:

$\omega_{j, s+1}=\omega_{j, s}(1-r)+r \frac{\pi_{j}}{\theta_{j}}$.

As previously mentioned, the score for heuristic $j$ obtained during the last segment $s$ is denoted as $\pi_{j}$. The number of times a heuristic $j$ has been used during the last segment $s$ is denoted as $\theta_{j}$. The reaction factor $r$ is used to control how much the roulette weight depends on the previous performance of heuristics. That is, if $r$ is equal to zero, the scores are not used to update the roulette weights; otherwise, the scores obtained in the last segment influence the roulette weights (Pisinger \& Ropke, 2010).

\section{Computational results}

In this section, we first explain the construction of problem instances, then the results of a parameter setting. In the last subsection, we present the computational experiments carried out with the goal of assessing the performance of the CSPP model and the LNS and ALNS approaches. The computer where the computational experiments were conducted is equipped with an Intel Xeon E5$26673.3 \mathrm{GHz}$ and $128 \mathrm{~GB}$ of RAM. The executions were limited to use only one CPU. The proposed LNS and ALNS approaches have been implemented in JAVA.

\subsection{Data and problem instances}

The assessment of the proposed mathematical model and large neighborhood approaches is performed on a set of well-defined problem instances. As explained in the following, we use real virtual machine type configurations provided by three leading cloud providers, whereas synthetic data is used to generate demand profiles representing different consumer and application task requirements.

\subsubsection{Virtual machine types}

To represent the supply in form of real IaaS compute offerings, we use real data collected from the three leading IaaS cloud providers: AWS, Microsoft, and Google. ${ }^{3}$ As listed in Appendix B, each virtual machine type offers a fixed amount of GFlops, memory capacity (in GB), and disk capacity (in GB). The type of the attached virtual CPU has been used to determine the number of GFlops after consulting respective performance benchmarks (see, e.g., Fujitsu, 2014). Overall, we consider 49 real virtual machine types from the three leading cloud providers, which can be categorized into standard, compute, and memory. The precise price of each virtual machine type depends on the operating system and the region a consumer chooses for deploying application tasks. Herein, the consumer is able to choose between Linux and Windows. As depicted in Fig. 3, each cloud provider offers to deploy each virtual machine type in various locations of four different continents. Note that we consolidate locations with identical prices per region denoted with the suffix all. This results in a price matrix containing all on-demand prices per hour depending on the operating system and region.

\subsubsection{Demand profiles}

To represent different consumer and task profiles in terms of consumer and application task requirements, we generate synthetic data based on a random number generator taking into account different ranges of required processing, memory, and storage capacities (see Table 4). The ranges are determined in a way that they accommodate different standard, compute-intensive, and memory-intensive tasks, such as those reported in the cloud computing literature (Coutinho et al., 2013; Coutinho et al., 2015). Each task specifies the amount of GFlops, memory capacity, and storage capacity. Moreover, we incorporate consumer requirements by randomly defining the operating system and region requirement for each application task. As depicted in Fig. 3, we choose among five different regions in which the three cloud providers offer the different types of virtual machines as explained in Section 5.1.1. Moreover, execution time deadlines are defined in a way that the problem instances are feasible.

\subsubsection{Problem instances}

Based on the collected data, we generate small, medium, and large problem instances by varying the number of application tasks between $10,50,100,250,500$, and 1000 . Three problem instances per task type and size are created to ensure the generality of results, resulting in a set of 54 problem instances. ${ }^{4}$ As such, to the best of our knowledge, this is the first comprehensive

\footnotetext{
3 Amazon EC2: https://aws.amazon.com/ec2/instance-types/, Microsoft Azure: https://azure.microsoft.com/de-de/services/virtual-machines/, Google Compute Engine: https://cloud.google.com/compute/pricing. All data is from May 27, 2015. Note that prices and configurations may differ at other dates.

4 The problem instances can be downloaded from: https://github.com/elalla/ Cloud-Service-Purchasing-Problem .
} 


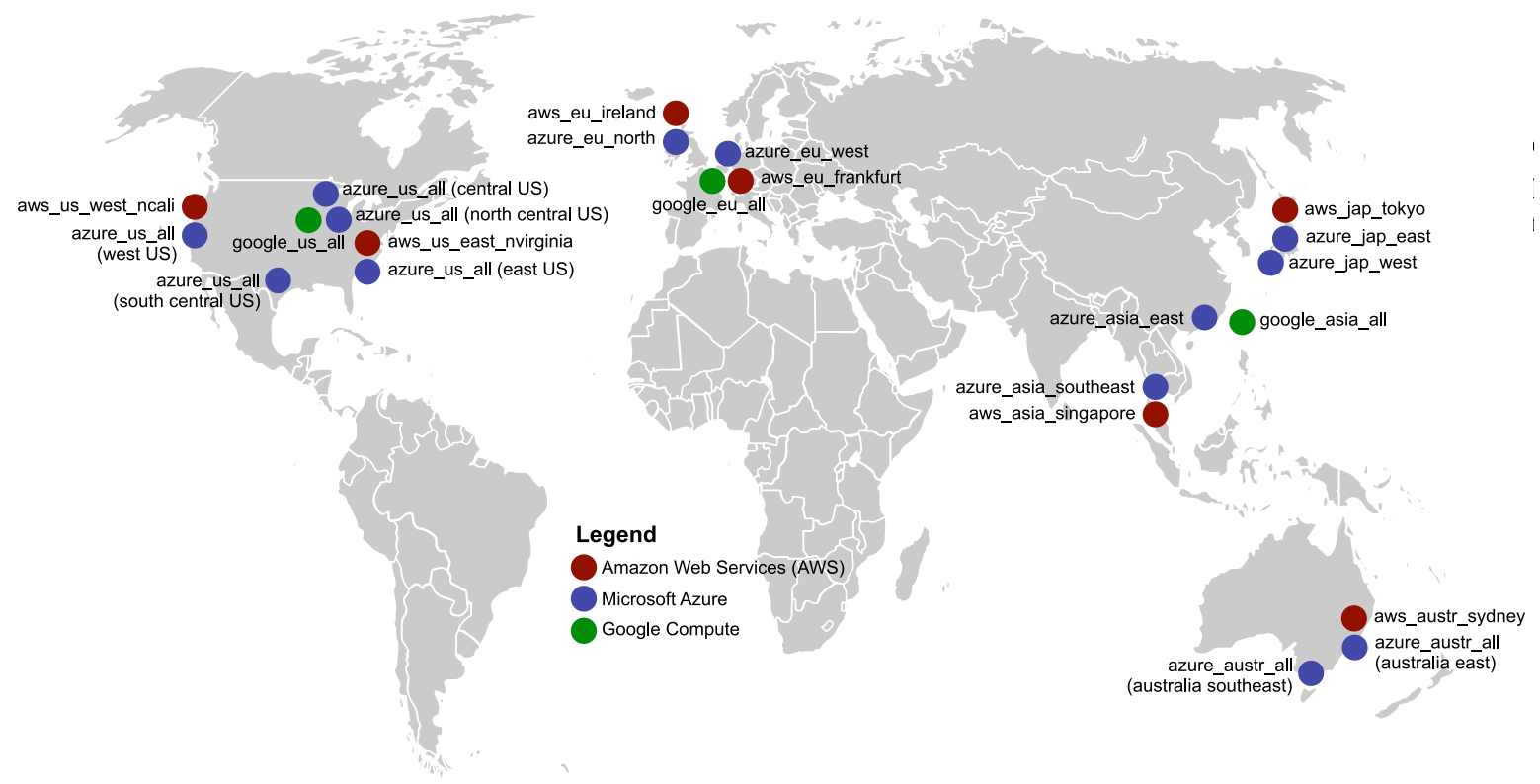

Fig. 3. Locations of available virtual machine types.

Table 4

Parameters for simulating different types of application tasks.

\begin{tabular}{llll}
\hline & Standard & Memory-intensive & Compute-intensive \\
\hline GFlop & $\{10080,604800\}$ & $\{10080,100800\}$ & $\{604800,43545600\}$ \\
Memory (GB) & $\{4,16\}$ & $\{16,256\}$ & $\{1,8\}$ \\
Storage (GB) & $\{100,1000\}$ & $\{100,1000\}$ & $\{100,1000\}$ \\
Operating system & & Linux, Windows $\{0,1\}$ \\
Region & & US, EU, Asia, Japan, Australia $\{1,5\}$ \\
\hline
\end{tabular}

Table 5

Parameter values used in the parameter setting

\begin{tabular}{ll}
\hline Parameter & Values \\
\hline$\xi$ & $\in\{0.4,0.5,0.6\}$ \\
seg $_{\text {iter }}$ & $\in\{200,300,400\}$ \\
$p$ & $\in\{3,4,5\}$ \\
$r$ & $\in\{0.5,0.6,0.7\}$ \\
Temp $_{\min }$ & $\in\{0.001,0.01,0.1\}$ \\
$\beta$ & $\in\{0.999,0.98,0.95\}$ \\
$\gamma$ & $\in\{0.001,0.01,0.1\}$ \\
\hline
\end{tabular}

benchmark set of problem instances for assessing cloud consumerrelated problems in the area of cloud computing.

\subsection{Parameter setting}

In order to determine the best parameters for our algorithms, we have conducted a proper selection of parameter values for the ALNS through a statistical analysis. For this purpose, reasonable parameter values to be assessed during the parameter setting are presented in Table 5. Once the performance of the ALNS for each combination of parameter values is obtained, as frequently applied in the literature (Birattari, Yuan, Balaprakash, \& Stützle, 2010; García, Molina, Lozano, \& Herrera, 2009; Pellegrini \& Birattari, 2006), we use the Friedman non-parametric statistical test (Daniel, 1990) to state an order of performances. The null hypothesis of this test states that the differences of test results result from randomness rather than by using different parameter settings. If the null hypothesis is rejected, the multiple comparisons test of Friedman can be used to determine the differences among combinations. To test the different parameters, we use a subset of representative prob- lem instances by randomly selecting two problem instances per problem size resulting in 12 representative problem instances. The same subset of problem instances is also used in Section 5.3.

According to the aforementioned discussion, the Friedman test is applied to the average objective function value reported by the ALNS on the 12 problem instances with 2187 different combinations of parameter values derived from Table 5 . In this case, the Friedman test rejects the hypothesis with a $\alpha_{\text {friedman }}=0.05$ significance level for the objective function values indicating that there are statistically significant differences among the combinations of parameter values. More specifically, the Friedman test result provides a performance ranking of the 2187 parameter configurations under analysis taking into account the objective function values obtained in different representative scenarios. Using this ranking, we select the best performing parameter setting: $\xi=0.4$, seg iter $=$ $300, p=4, r=0.7$, Temp $p_{\min }=0.001, \beta=0.999, \gamma=0.001$. Regarding the scores used in the LNS and ALNS, taking preliminary tests into account, we identified the following scores for finding new solutions: $\sigma_{1}=100, \sigma_{2}=25, \sigma_{3}=10$. This configuration of scores favors heuristics that lead to an improvement of current solutions and especially best solutions (see Section 4.5).

As it has been reported that the number of assignments removed from the current solution, referred to as the level of destruction $(\xi)$, has a considerable impact on the ALNS performance (Ribeiro \& Laporte, 2012; Ropke \& Pisinger, 2006), we further analyze the impact of $\xi$ on the solution quality and computational time. Table 6 presents the average improvement regarding the initial solution $\left(\operatorname{Impr}_{\text {init }}\right)$ in percentage as well as the average computational time in seconds ( $\mathrm{t}(\mathrm{s}$.)). It can be observed that the chosen destruction level $\xi=0.4$ provides the best solution quality. On average, it is plausible that the computational times correlate with the number of removals. 
Table 6

Impact of parameter $\xi$ on the solution quality and computational time tested with 12 representative problem instances using the average values of 15 executions.

\begin{tabular}{lllll}
\hline$\xi$ & 0.3 & 0.4 & 0.5 & 0.6 \\
Impr $_{\text {init }}$ & 7.55 & $\mathbf{7 . 7 1}$ & 7.27 & 6.77 \\
$\mathrm{t}(\mathrm{s})$. & 146.14 & $\mathbf{1 9 0 . 0 3}$ & 201.97 & 685.63 \\
\hline
\end{tabular}

Table 7

Comparison of destroy heuristics and the adaptive selection approach based on 12 representative problem instances using the average values of 15 executions.

\begin{tabular}{lllll}
\hline Destroy Heuristic & Shaw & Worst & Random & Adaptive \\
\hline Impr $_{\text {init }}$ & 6.68 & 7.23 & 6.63 & $\mathbf{7 . 7 1}$ \\
t (s.) & 286.00 & 93.55 & 104.02 & $\mathbf{1 9 0 . 0 3}$
\end{tabular}

In Appendix C, we further analyze the convergence of the ALNS in terms of the objective function value for the different values of parameter $\xi$. The plots depict the convergence of the ALNS along the execution time for different problem instances. In the depicted cases $\xi=0.4$ provides the best objective function value, except of one problem instance (Fig. C.10 (c)) where $\xi=0.3$ and $\xi=0.5$ provide slightly better values. In general, we see that the convergence of $\xi=0.4$ is satisfactory. It can be further observed that increasing the parameter $\xi$ from 0.4 results in a poorer convergence and higher computational times.

\subsection{Evaluation of destroy heuristics}

To further assess the performance of the adaptive selection of destroy heuristics (see Section 4.5), which is the core element of the proposed ALNS, we compare the performance of each destroy heuristic with the adaptive selection approach in Table 7. In the table, the average improvement with regards to the initial solution $\left(I m p r_{i n i t}\right)$ in percent as well as the average computational time in seconds ( $\mathrm{t}(\mathrm{s})$.$) are reported. We see that the adaptive selection,$ which incorporates all destroy heuristics, outperforms each individual destroy heuristic in terms of the average improvement of the initial solution. Concerning the computational time, it is logical that the adaptive selection remains between the average computational times of the three destroy heuristics.

\subsection{Results evaluation}

This subsection presents the computational experiments carried out with the goal of assessing the performance of the CSPP model and the large neighborhood approaches. In this regard, for ensuring a fair comparison, we executed all the experiments under the same CPLEX version (v12.6) within a time limit of 7200 seconds, CPLEX set to all-default and limited to 1 thread.

Tables 8 and 9 show the results provided by the mathematical model CSPP implemented in CPLEX, the initial solution provided by the greedy heuristic described in Section 4.2 (Greedy heuristic), the Large Neighborhood Search algorithm (LNS), and the Adaptive Large Neighborhood Search algorithm (ALNS). The column Instance contains the different problem instances described in Section 5.1. For each problem instance, under the column CPLEX we report the objective function value $(\mathrm{Obj}$.) as calculated in the mathematical model, the relative error provided by CPLEX (Gap (\%)), and the computational time $(t(s)$.$) . In the Greedy heuristic column we only$ report the objective function value $(\mathrm{Obj})$ due to the very short execution times of the related heuristic. Moreover, under the LNS and ALNS columns, we depict the average objective function value $(\mathrm{Obj})$ based on 10 executions, the error with respect to the best solution provided by CPLEX (Gap (\%)), the improvement with respect to the initial solution $\left(\operatorname{Impr}_{\text {init }}(\%)\right)$, number of iterations (Iter), and the computational time $(t(s)$.$) . It should be noted that in all the$ tables we rounded the results to two decimals. The gap is calculated using expression (28).

$\operatorname{Gap}(\%)=\frac{(U B-L B)}{U B} \cdot 100$,

Table 8

Computational results for the small- and medium-sized problem instances.

\begin{tabular}{|c|c|c|c|c|c|c|c|c|c|c|c|c|c|c|c|}
\hline \multirow[t]{2}{*}{ Nr. } & \multirow[t]{2}{*}{ Instance } & \multicolumn{3}{|l|}{ CPLEX } & \multirow{2}{*}{$\begin{array}{l}\text { Greedy } \\
\text { Heuristic } \\
\text { Obj }\end{array}$} & \multicolumn{5}{|l|}{ LNS } & \multicolumn{5}{|l|}{ ALNS } \\
\hline & & Obj & Gap (\%) & $\mathrm{t}(\mathrm{s})$. & & Obj & Gap (\%) & $I m p r_{i n i t}$ & Iter & $\mathrm{t}(\mathrm{s})$. & Obj & Gap (\%) & $\operatorname{Impr}_{\text {init }}(\%)$ & Iter & $\mathrm{t}(\mathrm{s})$. \\
\hline 1 & AWSGoogleAzure_10_compute_1 & 52.87 & 0.00 & 16.17 & 56.69 & 56.69 & 7.23 & 0.00 & 3501 & 0.64 & 56.69 & 7.23 & 0.00 & 1801 & 0.40 \\
\hline 2 & AWSGoogleAzure_10_compute_2 & 32.75 & 0.00 & 18.60 & 35.64 & 34.58 & 5.60 & 2.96 & 3506 & 0.63 & 34.58 & 5.60 & 2.96 & 1501 & 0.31 \\
\hline 3 & AWSGoogleAzure_10_compute_3 & 53.85 & 0.00 & 17.65 & 53.88 & 53.88 & 0.05 & 0.00 & 3501 & 0.64 & 53.88 & 0.05 & 0.00 & 1801 & 0.34 \\
\hline 4 & AWSGoogleAzure_10_memory_1 & 6.24 & 0.00 & 85.07 & 8.84 & 6.60 & 5.69 & 25.35 & 3510 & 0.63 & 6.71 & 7.53 & 24.05 & 2401 & 0.56 \\
\hline 5 & AWSGoogleAzure_10_memory_2 & 10.63 & 0.00 & 15.95 & 12.98 & 10.96 & 3.10 & 15.55 & 3506 & 0.59 & 10.96 & 3.10 & 15.55 & 2701 & 0.58 \\
\hline 6 & AWSGoogleAzure_10_memory_3 & 9.94 & 0.00 & 16.04 & 10.09 & 10.09 & 1.48 & 0.00 & 3501 & 0.62 & 10.09 & 1.48 & 0.00 & 2401 & 0.50 \\
\hline 7 & AWSGoogleAzure_10_standard_1 & 7.84 & 0.00 & 16.51 & 8.86 & 7.84 & 0.00 & 11.50 & 3510 & 0.63 & 8.38 & 6.92 & 5.37 & 2401 & 0.53 \\
\hline 8 & AWSGoogleAzure_10_standard_2 & 9.12 & 0.00 & 30.43 & 9.57 & 9.37 & 2.73 & 2.06 & 3503 & 0.61 & 9.37 & 2.73 & 2.06 & 2401 & 0.51 \\
\hline 9 & AWSGoogleAzure_10_standard_3 & 7.58 & 0.00 & 69.23 & 8.06 & 7.58 & 0.01 & 5.96 & 3506 & 0.63 & 7.58 & 0.01 & 5.96 & 2101 & 0.47 \\
\hline 10 & AWSGoogleAzure_50_compute_1 & 192.79 & 1.91 & 7200 & 215.58 & 212.83 & 10.40 & 1.27 & 4991 & 1.10 & 214.41 & 11.22 & 0.54 & 3301 & 0.57 \\
\hline 11 & AWSGoogleAzure_50_compute_2 & 228.78 & 0.01 & 7200 & 250.54 & 236.32 & 3.30 & 5.67 & 5815 & 1.27 & 240.13 & 4.96 & 4.15 & 3301 & 0.59 \\
\hline 12 & AWSGoogleAzure_50_compute_3 & 180.98 & 1.77 & 7200 & 187.88 & 180.48 & -0.28 & 3.94 & 6082 & 1.46 & 181.64 & 0.36 & 3.32 & 3001 & 0.59 \\
\hline 13 & AWSGoogleAzure_50_memory_1 & 36.69 & 66.85 & 7200 & 53.17 & 41.81 & 13.96 & 21.36 & 4387 & 1.00 & 42.01 & 14.49 & 21.00 & 4201 & 0.79 \\
\hline 14 & AWSGoogleAzure_50_memory_2 & 52.15 & 80.10 & 7200 & 49.94 & 34.64 & -33.58 & 30.64 & 6689 & 1.63 & 35.32 & -32.28 & 29.28 & 4201 & 0.91 \\
\hline 15 & AWSGoogleAzure_50_memory_3 & 47.13 & 78.71 & 7200 & 53.88 & 37.90 & -19.57 & 29.64 & 6191 & 1.34 & 38.00 & -19.37 & 29.46 & 4201 & 0.84 \\
\hline 16 & AWSGoogleAzure_50_standard_1 & 45.06 & 81.99 & 7200 & 40.19 & 32.84 & -27.13 & 18.30 & 6520 & 1.45 & 33.37 & -25.94 & 16.97 & 3901 & 0.74 \\
\hline 17 & AWSGoogleAzure_50_standard_2 & 35.62 & 72.63 & 7200 & 43.54 & 35.20 & -1.17 & 19.15 & 5615 & 1.35 & 35.41 & -0.60 & 18.69 & 3901 & 0.88 \\
\hline 18 & AWSGoogleAzure_50_standard_3 & 42.88 & 75.94 & 7200 & 45.19 & 36.75 & -14.30 & 18.69 & 4585 & 1.04 & 36.95 & -13.84 & 18.24 & 3901 & 0.73 \\
\hline 19 & AWSGoogleAzure_100_compute_1 & - & - & - & 393.61 & 376.79 & - & 4.27 & 8115 & 6.74 & 377.09 & - & 4.20 & 3901 & 1.92 \\
\hline 20 & AWSGoogleAzure_100_compute_2 & - & - & - & 405.76 & 391.53 & - & 3.51 & 8712 & 7.15 & 393.25 & - & 3.08 & 3901 & 1.87 \\
\hline 21 & AWSGoogleAzure_100_compute_3 & - & - & - & 421.74 & 403.53 & - & 4.32 & 9274 & 7.74 & 404.69 & - & 4.04 & 3901 & 2.14 \\
\hline 22 & AWSGoogleAzure_100_memory_1 & - & - & - & 102.99 & 73.63 & - & 28.51 & 7726 & 6.26 & 74.19 & - & 27.97 & 2401 & 1.62 \\
\hline 23 & AWSGoogleAzure_100_memory_2 & - & - & - & 100.05 & 75.13 & - & 24.91 & 8471 & 6.79 & 75.75 & - & 24.28 & 2401 & 1.42 \\
\hline 24 & AWSGoogleAzure_100_memory_3 & - & - & - & 95.07 & 66.90 & - & 29.63 & 7953 & 6.44 & 67.05 & - & 29.47 & 2401 & 1.50 \\
\hline 25 & AWSGoogleAzure_100_standard_1 & - & - & - & 82.46 & 66.97 & - & 18.78 & 8224 & 6.61 & 67.85 & - & 17.72 & 2401 & 1.59 \\
\hline 26 & AWSGoogleAzure_100_standard_2 & - & - & - & 85.35 & 69.89 & - & 18.11 & 10,206 & 8.21 & 70.68 & - & 17.18 & 2401 & 1.56 \\
\hline \multirow[t]{2}{*}{27} & AWSGoogleAzure_100_standard_3 & - & - & - & 84.65 & 72.28 & - & 14.62 & 8214 & 6.59 & 73.35 & - & 13.35 & 2401 & 1.45 \\
\hline & Average & - & - & - & 108.01 & 97.89 & - & 13.29 & 5900 & 2.95 & 98.49 & - & 12.55 & 2945.44 & 0.96 \\
\hline
\end{tabular}


Table 9

Computational results for the large-sized problem instances.

\begin{tabular}{|c|c|c|c|c|c|c|c|c|c|c|}
\hline \multirow[t]{2}{*}{$\mathrm{Nr}$} & \multirow[t]{2}{*}{ Instance } & \multirow{2}{*}{$\begin{array}{l}\text { Greedy Heuristic } \\
\text { Obj }\end{array}$} & \multicolumn{4}{|l|}{ LNS } & \multicolumn{4}{|l|}{ ALNS } \\
\hline & & & Obj & $I m p r_{\text {init }}(\%)$ & Iter & $\mathrm{t}(\mathrm{s})$. & Obj & Impr $_{\text {init }}$ & Iter & $\mathrm{t}(\mathrm{s})$. \\
\hline 28 & AWSGoogleAzure_250_compute_1 & 1077.58 & 1050.92 & 2.47 & 6528 & 43.19 & 1045.78 & 2.95 & 4801 & 16.02 \\
\hline 29 & AWSGoogleAzure_250_compute_2 & 1127.662 & 1081.22 & 4.12 & 7681 & 50.94 & 1079.49 & 4.27 & 4801 & 20.19 \\
\hline 30 & AWSGoogleAzure_250_compute_3 & 1033.47 & 1013.38 & 1.94 & 6759 & 45.09 & 1017.94 & 1.50 & 4801 & 15.56 \\
\hline 31 & AWSGoogleAzure_250_memory_1 & 254.35 & 180.89 & 28.88 & 7674 & 51.50 & 177.94 & 30.04 & 3301 & 17.96 \\
\hline 32 & AWSGoogleAzure_250_memory_2 & 260.26 & 181.81 & 30.14 & 7769 & 52.36 & 180.97 & 30.47 & 3301 & 18.16 \\
\hline 33 & AWSGoogleAzure_250_memory_3 & 261.141 & 190.25 & 27.15 & 8474 & 57.08 & 188.78 & 27.71 & 3301 & 16.04 \\
\hline 34 & AWSGoogleAzure_250_standard_1 & 233.407 & 191.93 & 17.77 & 7296 & 48.04 & 190.81 & 18.25 & 3301 & 16.86 \\
\hline 35 & AWSGoogleAzure_250_standard_2 & 246.305 & 199.77 & 18.89 & 6629 & 43.81 & 200.97 & 18.41 & 3301 & 17.03 \\
\hline 36 & AWSGoogleAzure_250_standard_3 & 213.238 & 170.01 & 20.27 & 7648 & 50.76 & 170.51 & 20.04 & 3301 & 18.79 \\
\hline 37 & AWSGoogleAzure_500_compute_1 & 2091.109 & 2066.70 & 1.17 & 5399 & 204.66 & 2040.89 & 2.40 & 5401 & 51.65 \\
\hline 38 & AWSGoogleAzure_500_compute_2 & 1996.943 & 1986.02 & 0.55 & 4559 & 171.94 & 1981.62 & 0.77 & 5401 & 72.03 \\
\hline 39 & AWSGoogleAzure_500_compute_3 & 2121.214 & 2106.30 & 0.70 & 5010 & 188.54 & 2083.91 & 1.76 & 5401 & 53.76 \\
\hline 40 & AWSGoogleAzure_500_memory_1 & 479.179 & 349.32 & 27.10 & 7975 & 306.39 & 348.58 & 27.26 & 3901 & 112.30 \\
\hline 41 & AWSGoogleAzure_500_memory_2 & 505.887 & 362.88 & 28.27 & 8328 & 323.41 & 359.45 & 28.95 & 4201 & 109.23 \\
\hline 42 & AWSGoogleAzure_500_memory_3 & 518.07 & 383.60 & 25.96 & 7579 & 292.74 & 380.89 & 26.48 & 4201 & 145.22 \\
\hline 43 & AWSGoogleAzure_500_standard_1 & 434.214 & 357.59 & 17.65 & 8606 & 330.45 & 359.47 & 17.21 & 3901 & 67.28 \\
\hline 44 & AWSGoogleAzure_500_standard_2 & 428.641 & 353.26 & 17.59 & 9416 & 360.33 & 355.21 & 17.13 & 3901 & 108.08 \\
\hline 45 & AWSGoogleAzure_500_standard_3 & 400.466 & 335.24 & 16.29 & 9075 & 344.94 & 337.70 & 15.67 & 3901 & 117.69 \\
\hline 46 & AWSGoogleAzure_1000_compute_1 & 4124.855 & 4124.85 & 0.00 & 3501 & 806.69 & 4093.58 & 0.76 & 6301 & 484.23 \\
\hline 47 & AWSGoogleAzure_1000_compute_2 & 4313.757 & 4302.38 & 0.26 & 4003 & 925.28 & 4255.86 & 1.34 & 6301 & 316.04 \\
\hline 48 & AWSGoogleAzure_1000_compute_3 & 4244.041 & 4242.40 & 0.04 & 3988 & 914.84 & 4190.72 & 1.26 & 6301 & 273.09 \\
\hline 49 & AWSGoogleAzure_1000_memory_1 & 1006.078 & 726.87 & 27.75 & 8012 & 1878.97 & 720.78 & 28.36 & 4801 & 410.45 \\
\hline 50 & AWSGoogleAzure_1000_memory_2 & 985.859 & 722.70 & 26.69 & 6988 & 1633.72 & 716.96 & 27.28 & 4801 & 506.18 \\
\hline 51 & AWSGoogleAzure_1000_memory_3 & 998.448 & 722.71 & 27.62 & 7959 & 1866.31 & 715.20 & 28.37 & 4801 & 1028.20 \\
\hline 52 & AWSGoogleAzure_1000_standard_1 & 907.345 & 739.21 & 18.53 & 6442 & 1492.69 & 741.39 & 18.29 & 4801 & 722.49 \\
\hline 53 & AWSGoogleAzure_1000_standard_2 & 853.874 & 702.15 & 17.77 & 8400 & 1950.92 & 704.32 & 17.51 & 4501 & 490.17 \\
\hline \multirow[t]{2}{*}{54} & AWSGoogleAzure_1000_standard_3 & 899.52 & 729.04 & 18.95 & 5577 & 1294.11 & 728.09 & 19.06 & 4501 & 941.98 \\
\hline & Average & 1185.81 & 1095.31 & 15.72 & 6936 & 582.58 & 1087.70 & 16.05 & 4501 & 228.40 \\
\hline
\end{tabular}

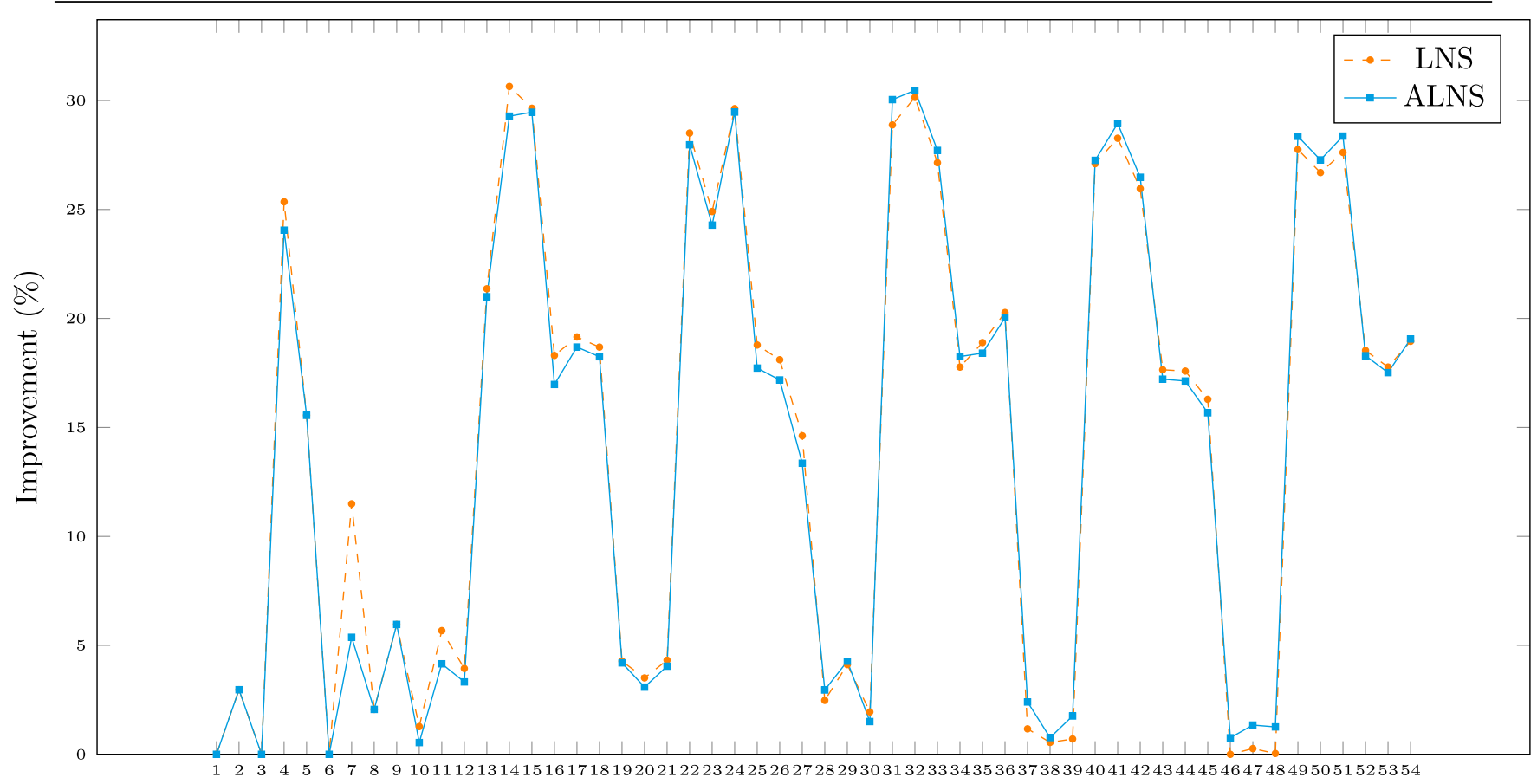

Problem instance

Fig. 4. Cost improvement in percent achieved by LNS and ALNS.

where $U B$ and $L B$ are the upper and lower bounds, respectively. The result is further visualized in Fig. 4 showing the improvement in percent compared to the initial greedy heuristic.

The results for the small- and medium-sized problem instances, as reported in Table 8, show that the mathematical formulation is only able to solve the small instances with 10 tasks to optimality. By means of those results, we are able to analyze the performance of our large neighborhood approaches. Their performance is highlighted when tackling larger instances. For case of problem instances with 50 tasks, a feasible solution can be provided only with a gap of $51 \%$ on average since CPLEX reaches the given time limit. For the problem instances with 100 tasks and more, CPLEX runs out of memory. It is therefore advisable to use of metaheuristics for obtaining a feasible solution in a reasonable time as well as the inclusion of the initial solution to measure the improvement obtained through such methods. In this context, as can be checked in the table, the LNS and ALNS are able to provide a feasible solution in all the cases within less than 3 seconds. 

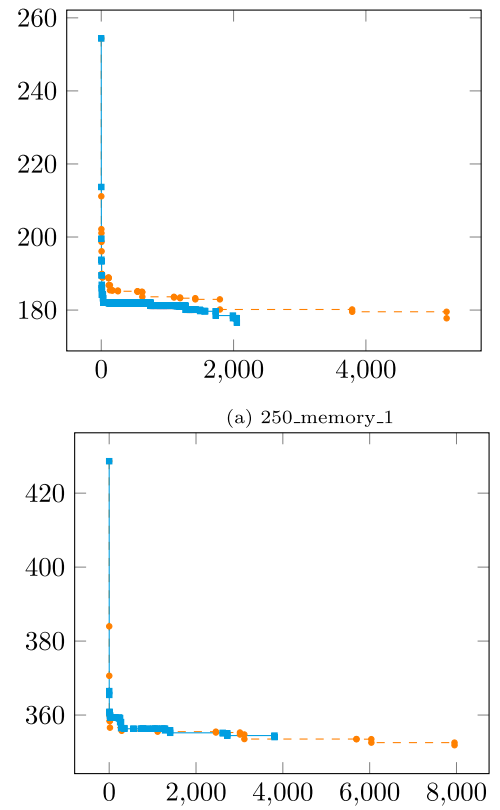

(c) 500_standard_2

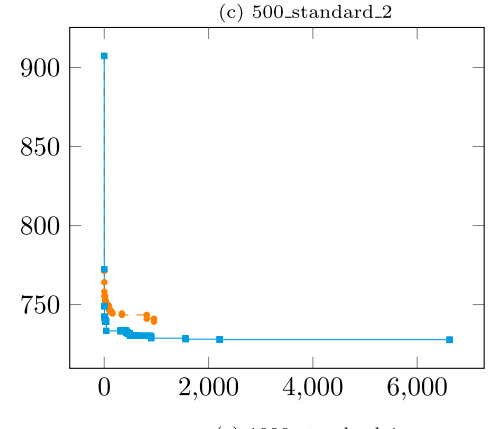

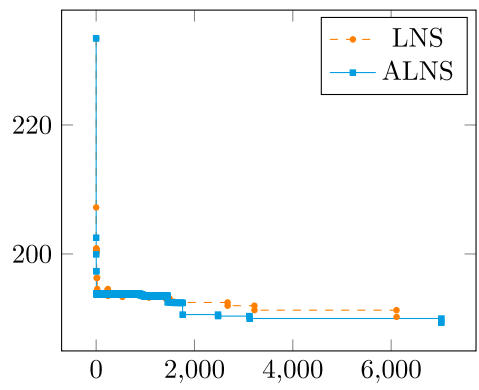

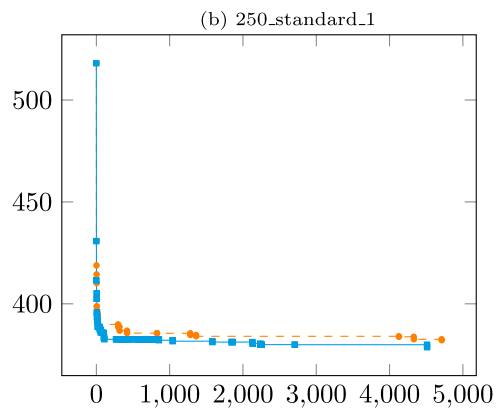

(d) 500_memory_3

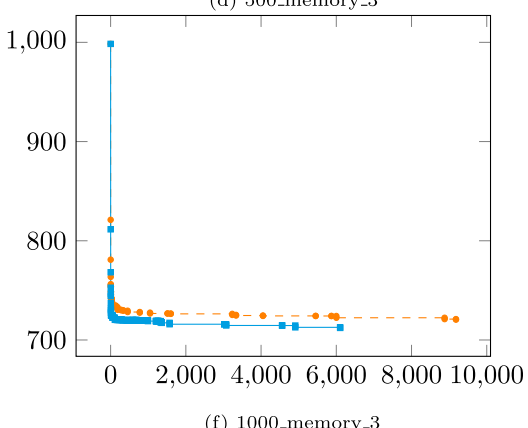

Fig. 5. Analysis of the convergence of LNS and ALNS.

Although both approaches have a gap of $2.88 \%$ and $3.85 \%$ for the 10 task problem instances on average, respectively, the time difference is noticeable. In contrast, concerning the instances with 50 tasks, the LNS and ALNS improve the solution quality with respect to CPLEX by an average of $7.60 \%$ and $6.78 \%$, respectively. In this regard, it is worth highlighting that the time performance of both large neighborhood approaches remains very competitive when the instance size is increased, especially if we compare it to the execution time required by CPLEX. Concerning the comparison between the large neighborhood approaches, we can remark that ALNS performs slightly worse than the LNS in terms of the relative improvement over all tested problem instances.

Comparing the results to the initial solution provided by a greedy heuristic (see Section 4.2), LNS improves the initial solution by $14.50 \%$, while ALNS exhibits an improvement of $14.30 \%$ on average. However, we see that ALNS outperforms LNS with respect to medium-size and large-size instances, shown in Table 9, where the results of ALNS are $2.11 \%$ better on average. This results in the fact that ALNS outperforms LNS over all tested problem instances in terms of the absolute cost improvement. While the absolute cost improvement of ALNS is 2905.90 (\$), using LNS costs 189.25 (\$) more. As it can also be seen in Fig. 4, ALNS provides in 28 out of 54 problem instances the same or a better objective function value compared to LNS.

By analyzing the extent of improvement with respect to the different problem instance types in Fig. 4, a recurring pattern can be recognized. That is, problem instances with standard application tasks provide the highest potential for improvement
( $25.29 \%$ with LNS and $25.33 \%$ with ALNS, on average), whereas ones with compute-intensive application tasks provide the lowest potential ( $2.07 \%$ with LNS, $2.18 \%$ with ALNS, on average). This is mostly related to the reduction of the number of virtual machines: the number of virtual machines used after optimizing can be most reduced for memory-intensive problem instances $(39.21 \%)$ compared to standard (33.60\%) and memory ones (25.21\%). However, it should be also noted that the percentage improvement relates to the unnormalized objective function values; in absolute terms, we see that the difference of the average cost improvement among the different problem instance types is not considerable large.

Regarding the computational time, the time improvement exhibited by ALNS is remarkable. Over all instances, ALNS needs only $39.17 \%$ of the computational time to achieve a similar solution quality compared to LNS. This especially holds true when the size of the instances strongly increases as it is the case in the large-size instance set, shown in Table 9. While ALNS solves the medium-size and large-size instances in less than 4 minutes, LNS almost needs $10 \mathrm{~min}$, on average. Therefore, it can be seen that the adaptive selection of heuristics leads to a considerable improvement of computational times while achieving competitive solutions. This favorable performance can also be seen in the convergence plots for large problem instances. In Fig. 5, the convergence of randomly chosen problem instances with a large number of standard and memory-intensive application is presented. The lines represent the best search execution in terms of best solution out of 15 executions, leading to the best objective value. As can be seen from the 

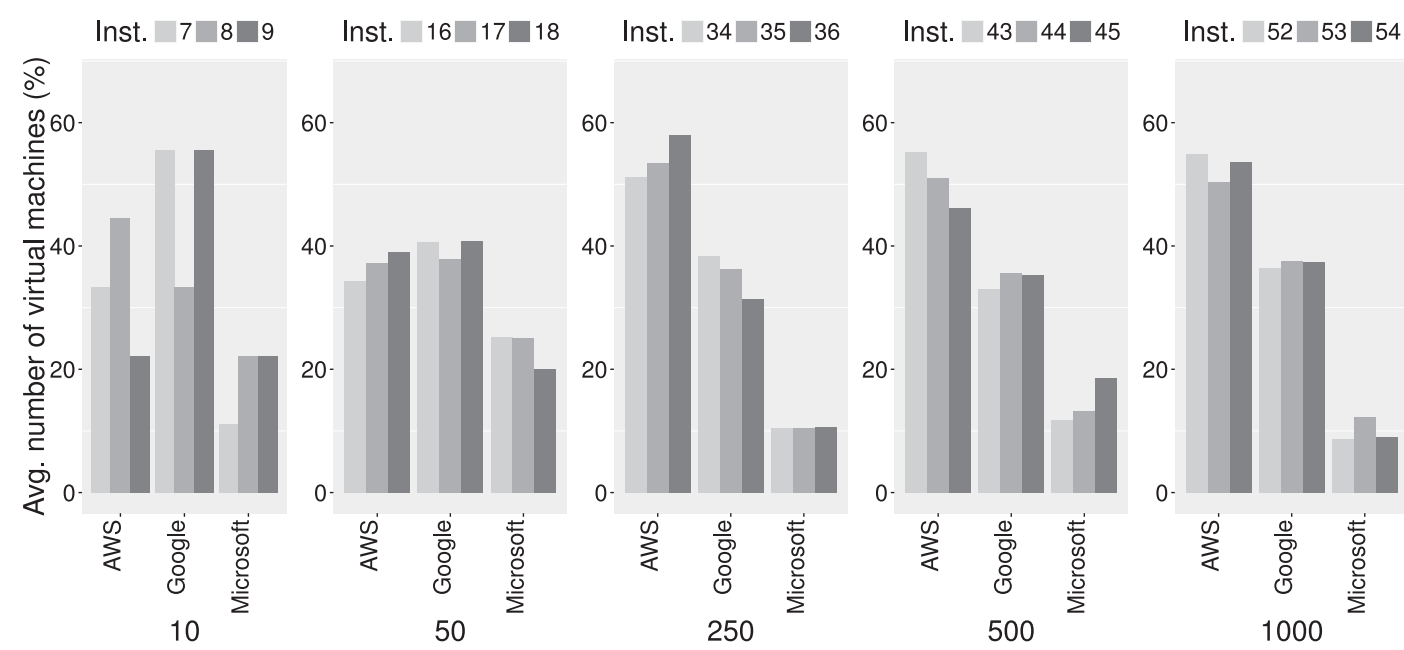

(a) Standard applications

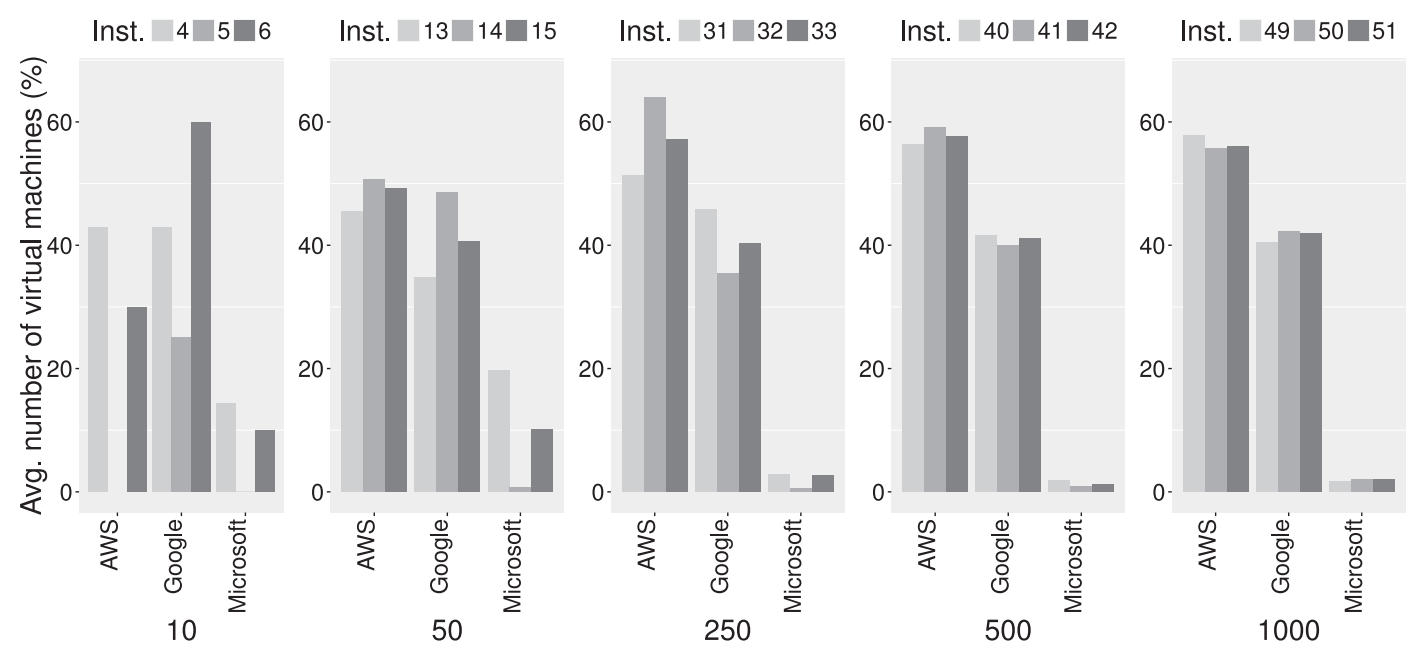

(b) Memory-intensive applications
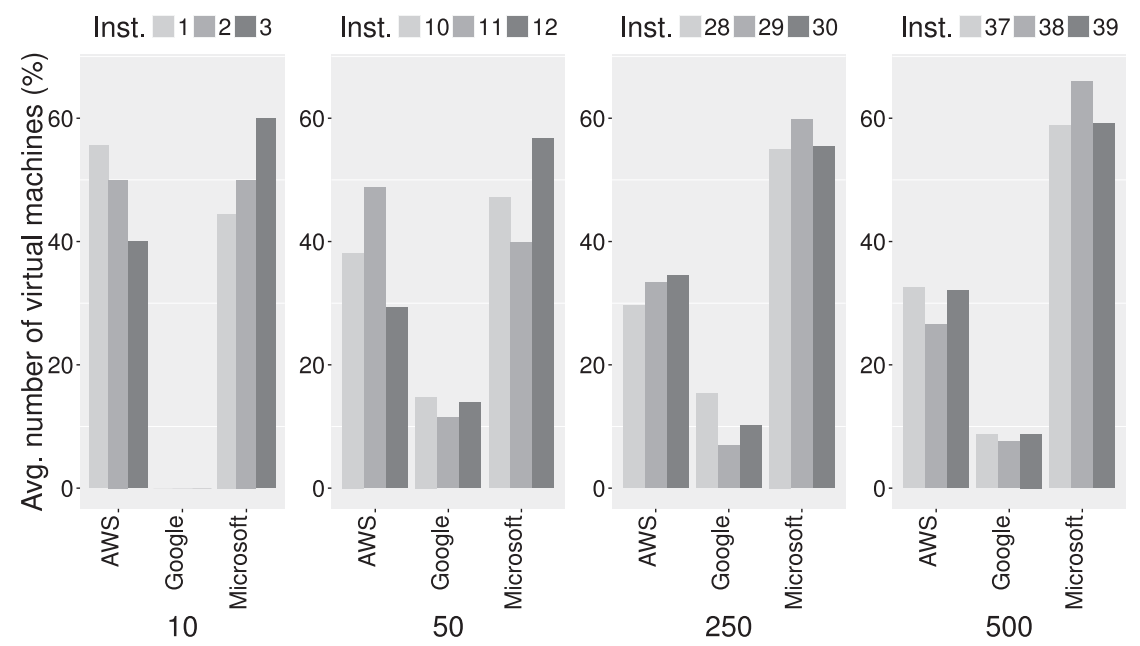

Inst. $46 \square 47 \square 48$

(c) Compute-intensive applications

Fig. 6. Usage of VMs among cloud providers. 
plots for most of the cases, the search of the ALNS finishes in less iterations and leads to a better objective function value. Note that the average computational time of one iteration in ALNS is, on average, lower compared to LNS. Therefore, it can be shown that the ALNS is more suitable for solving the CSPP, in particular for scenarios with a large number of application tasks.

\section{Comparison of cloud providers and usage patterns}

The proposed algorithms provide a fast and individual decision support for cloud brokers leading to significant cost reductions for cloud consumers. In particular for multi-cloud environments, this helps to better assess and understand the suitability of cloud providers and services alternatives. In this section, we use the results of our ALNS algorithm to analyze and compare the selection of cloud providers and services with respect to the three different application types (see Table 4).

\subsection{Cloud provider selection}

First, we analyze the distribution of applications among the three different cloud providers. As indicated, all of those cloud providers offer a wide range of on-demand virtual machine types for different prices, provisioned for standard, memory-intensive, and compute-intensive applications. Therefore, it is interesting to assess the strengths and weaknesses of cloud providers for specific application profiles. In this regard, the results provide interesting insights, which are depicted in Fig. 6.

- AWS: The largest share of applications, on average $45.48 \%$, is assigned to Amazon EC2 virtual machines. The results indicate that AWS provides in many cases the best alternative across all application types. For standard and memory-intensive applications, it provides in most of the cases the best alternative. Regarding compute-intensive applications, however, AWS faces a strong competition from Microsoft and therefore does not represent the best alternative in terms of costs.

- Google: The second largest share of applications, on average 29.47\%, is assigned to Google Compute Engine virtual machines. From our sample, it can be deduced that Google provides a good alternative for many standard and memory-intensive applications, but faces a strong competition from AWS. For compute-intensive applications, however, the results strongly indicate that Google is the best alternative for only a few applications. In small scenarios with ten applications, virtual machines of Google were not even used once.

- Microsoft: The amount of Microsoft Azure virtual machine assignments is close to the one of Google, on average at $25.05 \%$. The results indicate that Microsoft is particularly strong in providing economic virtual machine types for compute-intensive applications. For standard applications, Microsoft faces strong competition from Google and AWS. Especially for memoryintensive applications, only a very small amount of virtual machines from Microsoft is used to host those applications.

Overall, the results of our experiments accurately reflect potential differences in cloud service offerings among different cloud providers. This further emphasizes the importance of the proposed cloud brokerage mechanism in assessing and exploiting those differences in order to gain benefits for the consumer as long as all individual user and governance requirements are fulfilled. Rather than providing a tool for the strategic selection of cloud providers (see, e.g., Khajeh-Hosseini, Greenwood, Smith, \& Sommerville, 2012), the algorithms support operational decision making once cloud consumers have agreed relationships with a set of cloud providers. On the other hand, the results can be used by

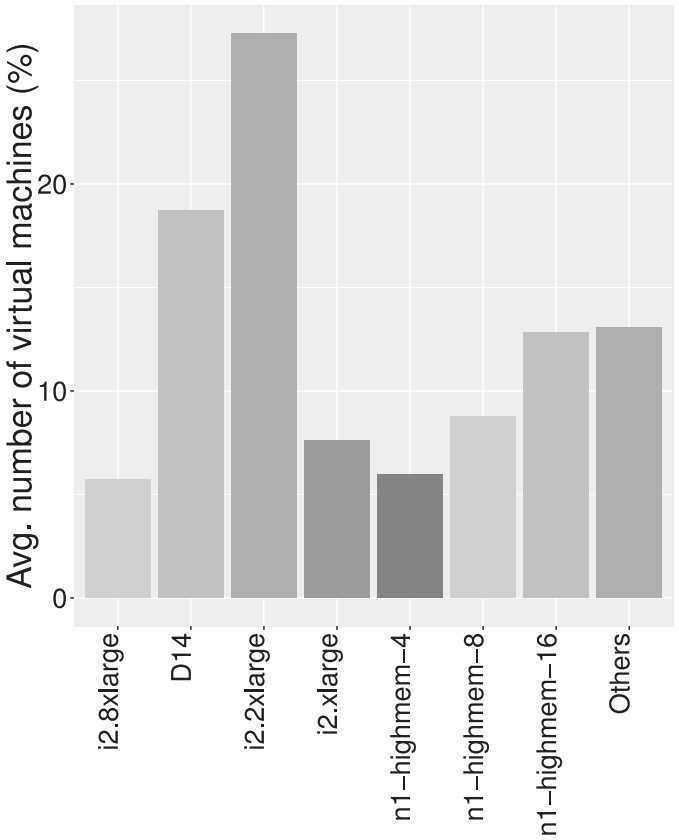

Fig. 7. Overall virtual machine type usage.

cloud providers as an input for strategic decisions with regard to the offered cloud service portfolio.

\subsection{Usage patterns of virtual machine types}

Next, we investigate the usage patterns related to specific virtual machine types of cloud providers. In particular, it is interesting to analyze if the different application types match with virtual machine types.

As depicted in Fig. 7, the results strongly indicate that virtual machine types with a high capacity in terms of all resource requirements are predominantly selected. Although these types of virtual machines are more expensive per hour of use, they provide some advantages in accommodating different types of applications in terms of sharing, performance, and overall costs. Nevertheless, a large number of virtual machines, on average $13.73 \%$, results from a variety of virtual machine types each contributing less than $5 \%$ of virtual machines.

Taking into account the application types, the results also depict the aforementioned tendency to select virtual machine types with large computing capacities (see Fig. 8). Another important observation we can make is that the strengths of individual cloud providers, analyzed in the previous subsection, mainly result from a few virtual machine types that are used for a large portion of applications. In comparison with AWS and Microsoft, a greater variety of virtual machine types of Google is used for accommodating applications in scenarios where they provide many good alternatives. We further see that the amount of other virtual machine types is especially low for standard and memory-intensive applications meaning that largely provisioned virtual machines provide more benefits. In terms of type matching, the results indicate that virtual machine types match the resource requirements of compute-intensive and memory-intensive application types. For compute-intensive applications, for example, virtual machine types with a large portion of GFlops are selected. This includes also virtual machines that are configured for memory-intensive applications, but also provide a large number of GFlops besides memory capacity. The reason for selecting more expensive types stems especially from the execution deadline of applications. For standard 

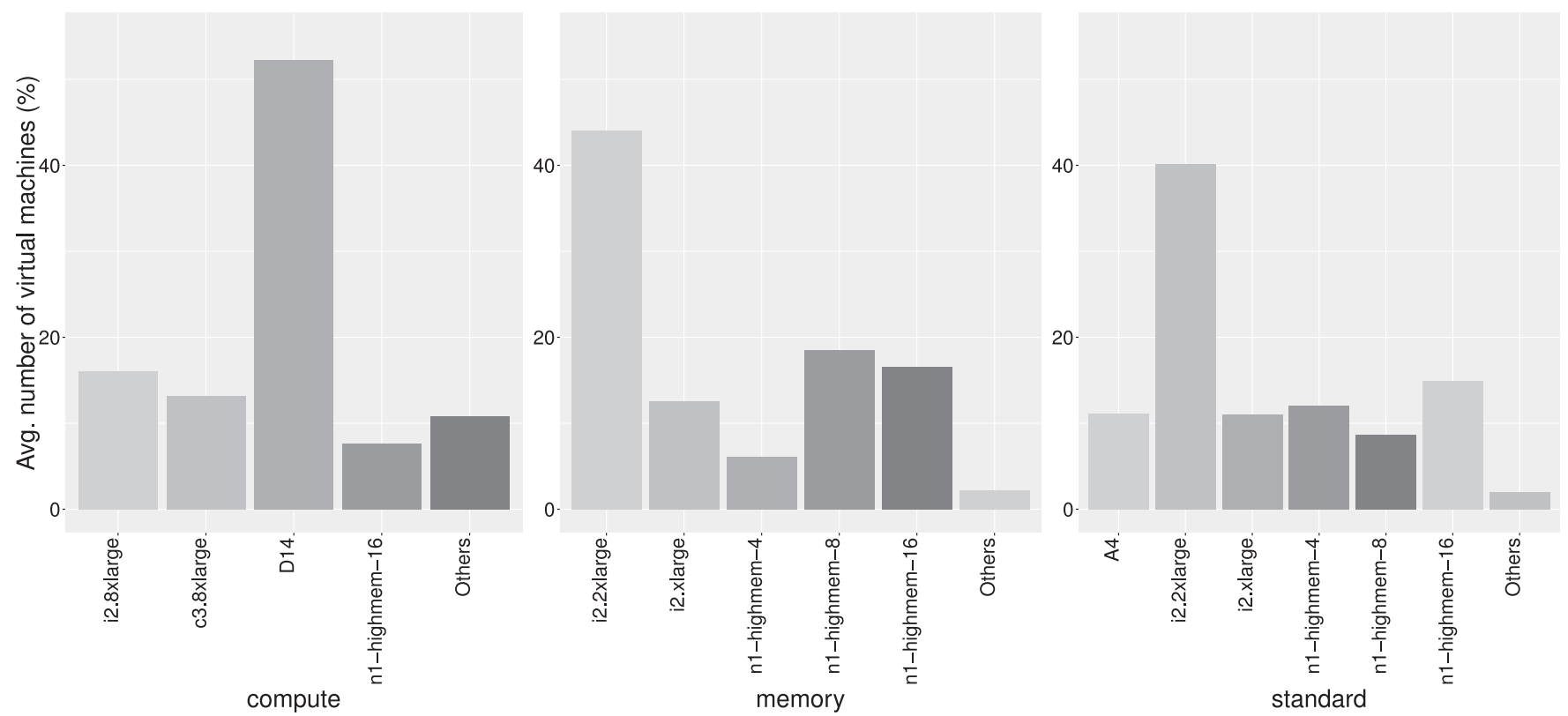

Fig. 8. Overall virtual machine type usage per application type.
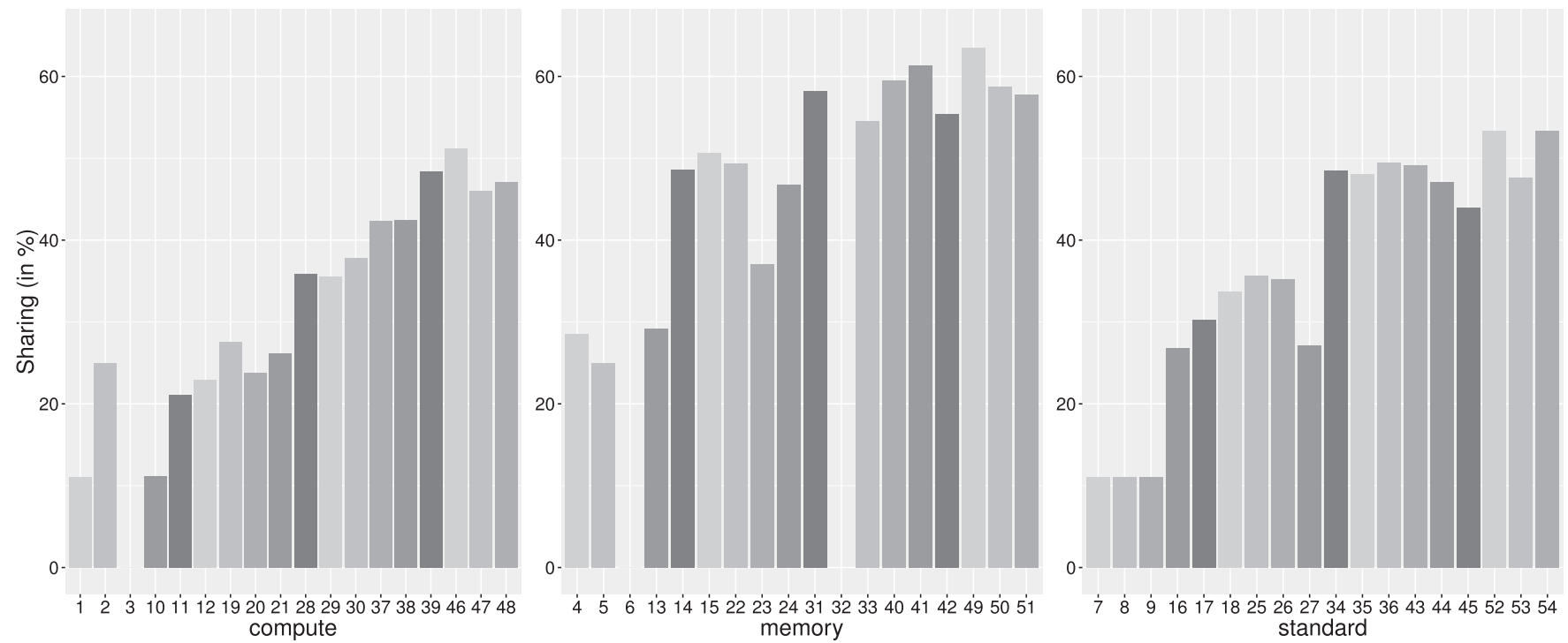

Fig. 9. Sharing of virtual machines.

applications, we observe again that largely provisioned virtual machine types in terms of GFlops, memory, and storage are selected.

\subsection{Sharing of virtual machines}

Finally, we analyze the sharing patterns of virtual machines among applications. In general, sharing virtual machines allows to drastically reduce costs. Given the computational results of the proposed algorithms, we see that the possibility of virtual machine sharing results in cost savings of about $22-23 \%$ on average. In Fig. 9, the amount of virtual machines hosting at least two applications is depicted. First of all, the results indicate a close relationship between the number of applications and the number of shared virtual machines. For scenarios with a small number of applications, it is more difficult, or even impossible (e.g., scenarios 3,6 ), to share virtual machines as the requirements of those application might vary. For example, it might be that they need to be executed in different locations using different operating systems. For scenarios with a large number of applications, it is easier to provision virtual machines satisfying the constraints of multiple applications. This allows to better utilize purchased virtual machines and leads to the aforementioned cost reductions. Regarding the different application types, we see that virtual machine sharing is most common for memory-intensive applications. Mostly used virtual machine types for those applications (see Section 6.2) are equipped with a large GFlop capacity such that more memoryintensive applications, requiring less GFlops than standard and compute-intensive applications (see Table 4), can be executed on a shared virtual machine within one time period. Thereby, purchased virtual machine hours can be utilized more economically.

\section{Concluding remarks and perspectives}

The application of operations research methods is increasingly gaining importance and interest in the area of cloud computing research. In this paper, we have proposed the Cloud Service Purchasing Problem (CSPP) devoted to supporting cloud brokerage for selecting cloud resources on behalf of cloud consumers by con- 
sidering essential consumer and application task requirements. The main goal in this work is to aid decision-making aimed at minimizing the costs of purchasing virtual machines hosting the application tasks of cloud consumers. As related works cover only certain aspects of this problem, we have proposed a novel mathematical model to solve the problem at hand. Moreover, since solving the CSPP exactly requires large amounts of computational time and memory, a Large Neighborhood Search (LNS) and an Adaptive Large Neighborhood Search (ALNS) have been implemented to obtain solutions in reasonable computational time. Using a large set of rich and well-defined problem instances, generated based on real data from leading cloud providers, the performance of the model and algorithms in terms of solution quality and computational time has been assessed.

The computational results have shown that our large neighborhood approaches are able to solve the CSPP within the range of seconds for the small-sized instances and up to a few minutes for the medium- and large-sized ones. While the optimization model implemented on a general-purpose solver such as CPLEX is not even able to reach feasible solutions for the medium and large cases within a time limit of two hours, both, LNS and ALNS exhibit a high competitiveness. It can be demonstrated that applying ALNS leads to considerable improvements regarding the convergence and computational time, while the relative improvement over all problem instances is similar to the one provided by the LNS algorithm. In comparison, ALNS performs better on medium- and large-sized problem instances and therefore leads to higher absolute cost improvements. Furthermore, the results support the strategy of sharing virtual machines among tasks as a way to reduce costs and resources. These advantages allow, on the brokerage side, to provide integrative technological solutions that efficiently use the acquired virtual machines, resulting in economic benefits for cloud consumers. As a result of the short computational times, the ALNS approach particularly suits the requirements of automated deployment processes as discussed in Heilig et al. (2015), necessary to address shifting demands and utilize the agile characteristics of cloud computing environments.

Moreover, results are investigated from a business perspective by comparing cloud providers and analyzing usage and sharing patterns using various scenarios with standard, memory-intensive, and compute-intensive types of application tasks. Thereby, important differences between cloud providers and aspects regarding the selection and sharing of virtual machine types are unveiled, for instance, strengths and weaknesses of cloud providers in providing well-configured virtual machine types for different types of application tasks. We further see that huge economic benefits can be achieved by purchasing well-provisioned, more expensive virtual machine types rather than the cheaper less-provisioned alternatives. We also observe economies of scale resulting from deploying a huge number of tasks on shared virtual machines. Thus, the proposed method allows to benchmark different cloud providers and their virtual machine type offerings. On the other hand, it allows cloud providers to adapt their cloud service portfolio to better suit different types of tasks and to make pricing decisions. Finally, the contributions of this work constitutes a basis for the design and development of expert and intelligent systems for solving and supporting the decision-making in multi-cloud environments.

Based on the findings given in this work, as future research, we intend to extend our approach by following three research directions. First, we aim to assess other metaheuristics for this problem to further validate the effectiveness of LNS and ALNS for the CSPP. Second, we aim to tackle the problem as a multi-objective optimization problem. This may involve minimizing the time needed to execute tasks as well as the distance of cloud locations to the consumers' location or the distance of tasks to each other in order to lower latency and bandwidth costs. For investigating the lat- ter, we intend to model interdependencies between tasks in workflow applications and corresponding data flows between clouds and virtual machines. Incorporating means of predictive analytics and simulations for estimating consumer demand patterns and a varying performance of cloud resources is regarded as another important aspect. Finally, we intend to explicitly extend our rather generic model for addressing certain operational requirements regarding very specific applications involving dynamism, such as real-time virtual machine deployment, big data architecture, etc.

\section{Declaration of Competing Interest}

The authors whose names are listed immediately below certify that they have NO affiliations with or involvement in any organization or entity with any financial interest (such as honoraria; educational grants; participation in speakers' bureaus; membership, employment, consultancies, stock ownership, or other equity interest; and expert testimony or patent-licensing arrangements), or nonfinancial interest (such as personal or professional relationships, affiliations, knowledge or beliefs) in the subject matter or materials discussed in this manuscript.

\section{Credit authorship contribution statement}

Leonard Heilig: Conceptualization, Data curation, Formal analysis, Validation, Writing - original draft. Eduardo Lalla-Ruiz: Conceptualization, Data curation, Formal analysis, Validation, Writing original draft. Stefan Voß: Conceptualization, Data curation, Formal analysis, Validation, Writing - original draft.

\section{Acknowledgments}

The authors want to thank the referees for constructive suggestions and comments provided for the revision of the manuscript.

\section{Appendix A. Adaptive large neighborhood search}

In this appendix, we explain the pseudocode version of the ALNS (Algorithm 4 ) presented in Section 4.1. The parameters used in the ALNS are the level of destruction in percent $(\xi)$, score increments $\left(\sigma_{1}, \sigma_{2}, \sigma_{3}\right)$ and a reaction factor $(r)$. The level of destruction determines how many assignments are removed from the current solution by the destroy heuristic. The maximum number of consecutive iterations in an execution segment $\left(\operatorname{seg}_{\max }\right)$, score increments and a reaction factor are required for applying the adaptive weight adjustment (see Section 4.5). Moreover, to incorporate simulated annealing, as it has been successfully done for other ALNS approaches (Pisinger \& Ropke, 2007; 2010), we need to incorporate additional parameters. The factor $\gamma$ is used to define the starting temperature based on the quality of the initial solution (line 6). Moreover, a stopping temperature $\left(T e m p_{\min }\right)$ and a cooling rate $(\beta)$ need to be provided.

The initial solution is generated (line 1 ) by using a constructive heuristic (see Section 4.2) and added to a set $K$ storing all accepted solutions (line 2). For applying the adaptive weight adjustment technique, a vector of roulette weights $\Omega=$ $\left[\omega_{1}, \omega_{2}, \ldots, \omega_{j}, \ldots, \omega_{|H|}\right]$ is initialized in line 5. For measuring the performance of the heuristics during the search, a score vector $\Pi=$ $\left[\pi_{1}, \pi_{2}, \ldots, \pi_{j}, \ldots, \pi_{|H|}\right]$ is initialized in line 8 . Moreover, a vector $\Theta=\left[\theta_{1}, \theta_{2}, \ldots, \theta_{j}, \ldots, \theta_{|H|}\right]$, for counting the number of times heuristic $j \in H$ is applied, is initialized in line 9 .

At each iteration, one destroy heuristic and one repair heuristic from the set $H$ are chosen by applying roulette wheel selection with the obtained roulette weights (line 12). After both destroy and repair heuristic have been chosen, vector $\Theta$ is updated. In line 


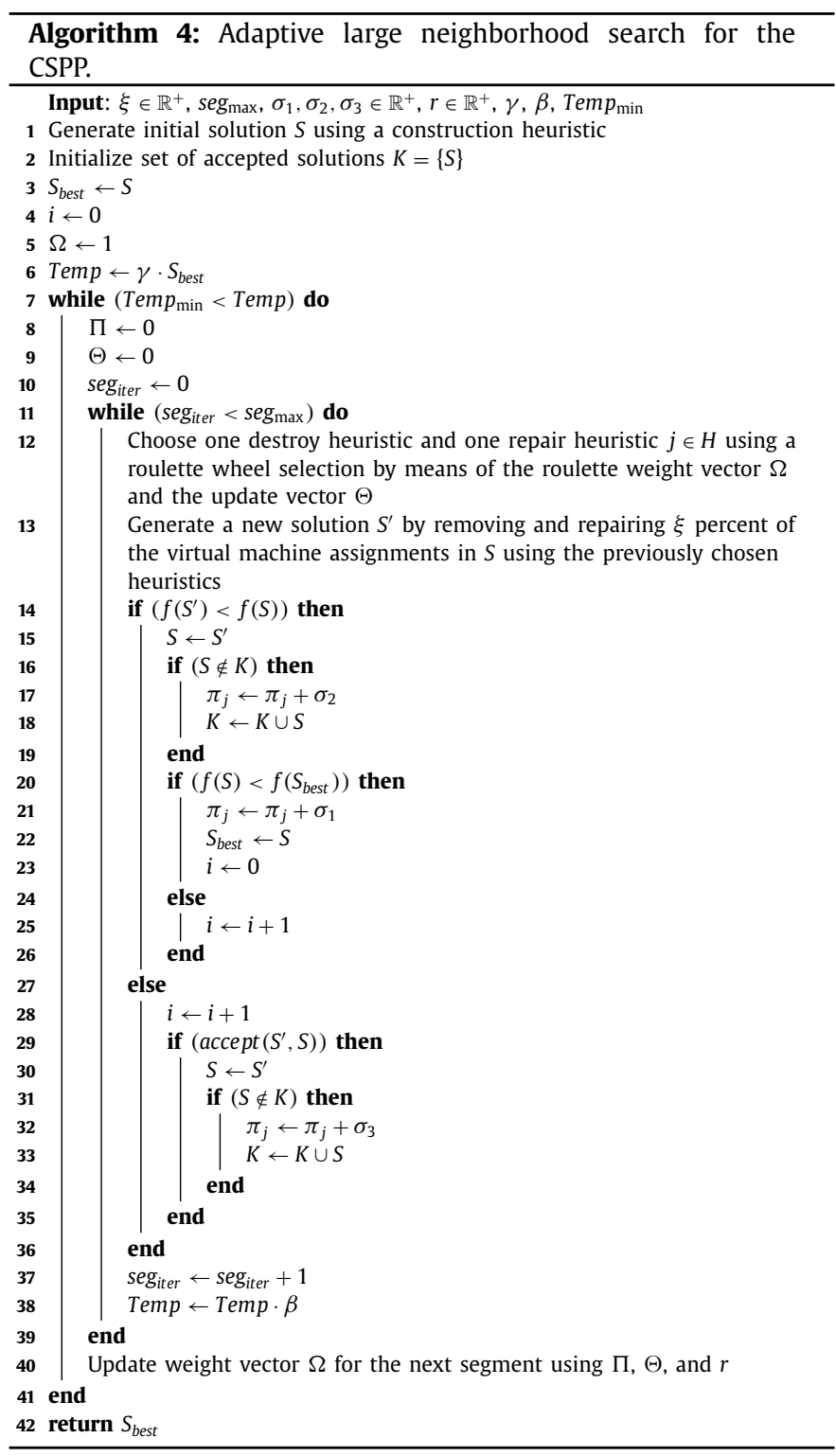

13 , a new solution $S^{\prime}$ is generated by alternately applying the selected destroy and repair heuristic to the current solution $S$ (see Section 4.4). The chosen destroy heuristic first selects assignments, linking an application task $a$ to a virtual machine $v$, which are removed from the current solution. The parameter $\xi=0.4$ (as determined in Section 5.2) determines the portion of assignments to be removed. A new solution is created by applying the repair heuristic assigning virtual machines to the unassigned applications. The objective function value is calculated by using function $f(S)$ (line $14,20)$. If the new solution is better than the current one in terms of objective function value, then the current solution is replaced by the one allowing an improvement (line 15). In case the updated current solution provides a new best solution value, the best known solution is also updated (line 22).

The acceptance function accept(·) allows to accept solutions that are worse than the current solution given an acceptance criterion (line 29). As stated before, we implement a simulated annealing acceptance criterion. Instead of accepting only solutions that are better than the current solution, the basic idea is to occasionally select worse solutions in order to avoid being trapped in a local minimum. Following the idea of simulated annealing, a worse solution may be accepted according to a probability that depends on the deterioration $\Delta$ of the objective function value. The probability is computed as $e^{\left(-\frac{\Delta}{T e m p}\right)}$, where Temp is used as a control parameter. The starting temperature is defined in line 6 based on the quality of the initial solution. At each iteration of a segment, the temperature is decreased Temp $\leftarrow$ Temp. $\beta$ (line 38), where $\beta$ denotes the cooling rate such that $0<\beta<1$.

Moreover, if applicable, the heuristic score $\pi_{j}$ of heuristic $j \in H$ in vector $\Pi$ is updated in lines 17,21 , and 32 . After a given number of iterations $\operatorname{seg}_{\text {iter, }}$, new roulette weights are calculated using the respective scores, $\pi_{j} \in \Pi$, vector $\Theta$, and the reaction factor $r$ (line 40). The latter parameter determines to which extent the previous weights and new scores of heuristics are taken into account in the calculation of new weights (see Section 4.5). The best solution obtained so far, $S_{\text {best }}$, is provided in line 42 after the temperature has reached a certain threshold, $T e m p_{\min }$ (line 7 ).

\section{Appendix B. Virtual machine types}

Table B1

Configurations of available virtual machine types.

\begin{tabular}{|c|c|c|c|c|c|c|}
\hline \multirow[b]{2}{*}{ AWS } & \multicolumn{6}{|c|}{ Package name } \\
\hline & $\begin{array}{l}\text { m3. } \\
\text { medium }\end{array}$ & m3.large & m3.xlarge & m3.2xlarge & c3.large & c3.xlarge \\
\hline GFlops & 190 & 380 & 760 & 1520 & 426 & 852 \\
\hline $\begin{array}{l}\text { Memory } \\
\text { (GB) }\end{array}$ & 3.75 & 7.5 & 15 & 30 & 3.75 & 7.5 \\
\hline \multirow[t]{2}{*}{ Disk (GB) } & 4 & 32 & 80 & 160 & 32 & 80 \\
\hline & c3.2xlarge & c3.4xlarge & c3.8xlarge & r3.large & r3.xlarge & r3.2xlarge \\
\hline GFlops & 1704 & 3408 & 6816 & 380 & 760 & 1520 \\
\hline $\begin{array}{l}\text { Memory } \\
\text { (GB) }\end{array}$ & 15 & 30 & 60 & 15.25 & 30.5 & 61 \\
\hline \multirow[t]{2}{*}{ Disk (GB) } & 160 & 320 & 640 & 32 & 80 & 160 \\
\hline & r3.4xlarge & r3.8xlarge & i2.xlarge & i2.2xlarge & i2.4xlarge & i2.8xlarge \\
\hline GFlops & 3040 & 6080 & 760 & 1520 & 3040 & 6080 \\
\hline $\begin{array}{l}\text { Memory } \\
\text { (GB) }\end{array}$ & 122 & 244 & 30.5 & 61 & 122 & 244 \\
\hline Disk (GB) & 320 & 640 & 800 & 1600 & 3200 & 6400 \\
\hline Microsoft & A0 & $\mathrm{A} 1$ & A2 & A3 & A4 & A5 \\
\hline GFlops & 168 & 168 & 335 & 670 & 1340 & 670 \\
\hline $\begin{array}{l}\text { Memory } \\
\text { (GB) }\end{array}$ & 0.75 & 1.75 & 3.5 & 7 & 14 & 14 \\
\hline \multirow[t]{2}{*}{ Disk (GB) } & 20 & 70 & 135 & 285 & 605 & 135 \\
\hline & A6 & A7 & D1 & D2 & D3 & D4 \\
\hline GFlops & 1340 & 2680 & 335 & 670 & 1340 & 2680 \\
\hline $\begin{array}{l}\text { Memory } \\
\text { (GB) }\end{array}$ & 28 & 56 & 3.5 & 7 & 14 & 28 \\
\hline \multirow[t]{2}{*}{ Disk (GB) } & 285 & 605 & 50 & 100 & 200 & 400 \\
\hline & D11 & D12 & D13 & D14 & A10 & A11 \\
\hline GFlops & 670 & 1340 & 2680 & 5360 & 3040 & 6080 \\
\hline $\begin{array}{l}\text { Memory } \\
\text { (GB) }\end{array}$ & 14 & 28 & 56 & 112 & 56 & 112 \\
\hline Disk (GB) & 100 & 200 & 400 & 800 & 382 & 382 \\
\hline Google & n1-std-1 & n1-std-2 & n1-std-4 & n1-std-8 & n1-std-16 & n1-hcpu-2 \\
\hline GFlops & 159 & 317 & 634 & 1268 & 2536 & 317 \\
\hline $\begin{array}{l}\text { Memory } \\
\text { (GB) }\end{array}$ & 3.75 & 7.5 & 15 & 30 & 60 & 1.8 \\
\hline \multirow[t]{2}{*}{ Disk (GB) } & 100 & 250 & 500 & 750 & 1000 & 250 \\
\hline & n1-hcpu-4 & n1-hcpu-8 & $\begin{array}{l}\text { n1-hcpu- } \\
16\end{array}$ & $\begin{array}{l}\text { n1-hmem- } \\
2\end{array}$ & $\begin{array}{l}\text { n1-hmem- } \\
4\end{array}$ & $\begin{array}{l}\text { n1-hmem- } \\
8\end{array}$ \\
\hline GFlops & 634 & 1268 & 2536 & 317 & 634 & 1268 \\
\hline $\begin{array}{l}\text { Memory } \\
\text { (GB) }\end{array}$ & 3.6 & 7.2 & 14.4 & 13 & 26 & 52 \\
\hline \multirow[t]{2}{*}{ Disk (GB) } & 500 & 750 & 1000 & 250 & 500 & 750 \\
\hline & $\begin{array}{l}\text { n1-hmem- } \\
16\end{array}$ & & & & & \\
\hline GFlops & 2536 & & & & & \\
\hline $\begin{array}{l}\text { Memory } \\
\text { (GB) }\end{array}$ & 104 & & & & & \\
\hline Disk (GB) & 1000 & & & & & \\
\hline
\end{tabular}




\section{Appendix C. Convergence with different Neighborhood Sizes}
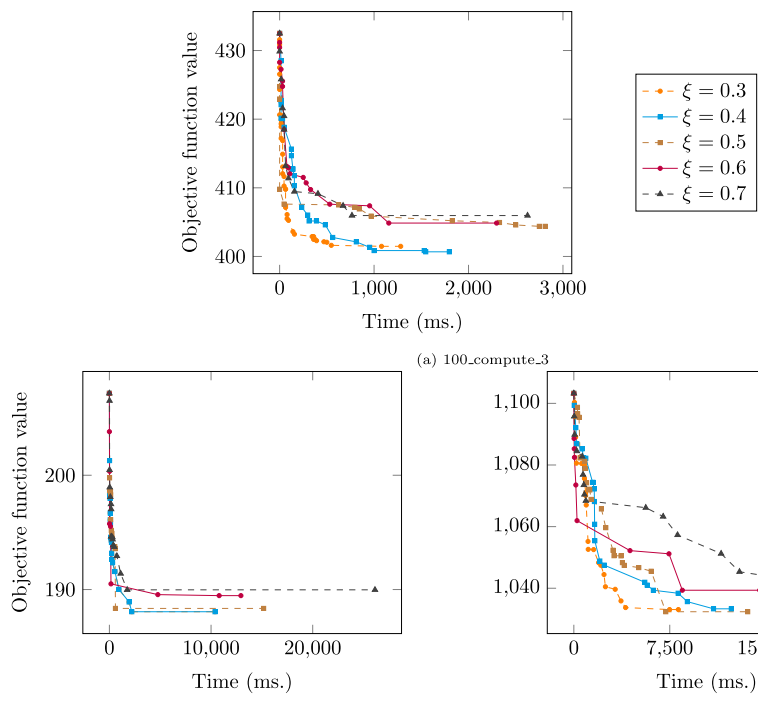

(a) 100_compute_3

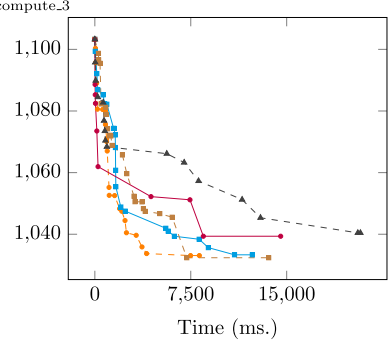

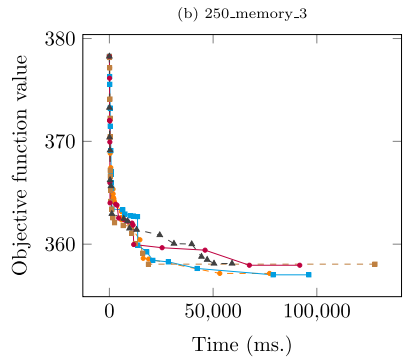

(d) 500_memory_2

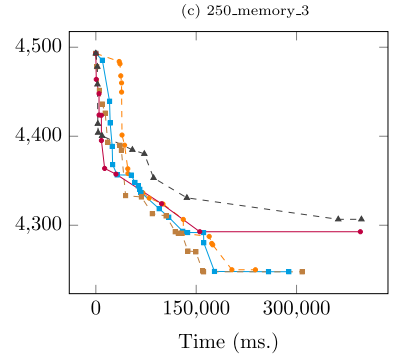

(e) 1000 compute_2
Fig. C1. Analysis of the convergence for different $\xi$ values.

\section{References}

Andriole, S. J. (2015). Who owns IT? Communications of the ACM, 58(3), 50-57. doi: $10.1145 / 2660765$.

Andrzejak, A., Kondo, D., \& Yi, S. (2010). Decision model for cloud computing under SLA constraints. In Proceedings of the IEEE international symposium on modeling, analysis $\mathcal{E}$ simulation of computer and telecommunication systems (mascots) (pp. 257-266). Miami Beach, FL: IEEE.

Armbrust, M., Fox, A., Griffith, R., Joseph, A. D., Katz, R., Konwinski, A., ... Zaharia, M. (2010). A view of cloud computing. Communications of the ACM, 53(4), 50-58.

AWS (2019). Using Amazon ECS parameters. https://docs.aws.amazon.com AmazonECS/latest/developerguide/cmd-ecs-cli-compose-ecsparams.html. Last accessed Dec 08, 2019.

Baldini, I., Castro, P., Chang, K., Cheng, P., Fink, S., Ishakian, V., ... Slominski, A., et al. (2017). Serverless computing: Current trends and open problems. In S. Chaudhary, G. Somani, \& R. Buyya (Eds.), Serverless computing: Current trends and open problems: Research Advances in Cloud Computing (pp. 1-20). Springer.

Bansal, N., Lee, K.-W., Nagarajan, V., \& Zafer, M. (2015). Minimum congestion mapping in a cloud. SIAM Journal on Computing, 44(3), 819-843.

Birattari, M., Yuan, Z., Balaprakash, P., \& Stützle, T. (2010). F-Race and iterated f-race: An overview. Experimental Methods for the Analysis of Optimization Algorithms, 311-336.

Van den Bossche, R., Vanmechelen, K., \& Broeckhove, J. (2010). Cost-optimal scheduling in hybrid laaS clouds for deadline constrained workloads. In Proceedings of the 3rd ieee international conference on cloud computing (cloud) (pp. 228-235). Miami, FL: IEEE.

Brandt, F., Speck, J., \& Völker, M. (2016). Constraint-based large neighborhood search for machine reassignment. Annals of Operations Research, 242(1), 63-91.

Buyya, R., Yeo, C. S., Venugopal, S., Broberg, J., \& Brandic, I. (2009). Cloud computing and emerging IT platforms: Vision, hype, and reality for delivering computing as the 5th utility. Future Generation Computer Systems, 25(6), 599-616.

Chaisiri, S., Lee, B.-S., \& Niyato, D. (2009). Optimal virtual machine placement across multiple cloud providers. In Proceedings of the 4th IEEE asia-pacific services computing conference (apscc) (pp. 103-110). Singapore

Chaisiri, S., Lee, B.-S., \& Niyato, D. (2012). Optimization of resource provisioning cost in cloud computing. IEEE Transactions on Services Computing, 5(2), 164-177.
Chanthadavong, A. (2014). Dell launches into cloud brokerage market with Cloud Marketplace. http://www.zdnet.com/article/ dell-launches-into-cloud-brokerage-market-with-cloud-marketplace. Last accessed Dec 08, 2019.

Chen, M., Mao, S., \& Liu, Y. (2014). Big data: a survey. Mobile Networks and Applications, 19(2), 171-209.

Chen, Y., Paxson, V., \& Katz, R. H. (2010). What's new about cloud computing security? Technical Report UCB/EECS-2010-5. University of California, Berkeley.

COSO (2012). Enterprise Risk Management for Cloud Computing. Technical Report. Committee of Sponsoring Organizations of the Treadway Commission (COSO).

Coutinho, R. d. C., Drummond, L. M. A., \& Frota, Y. (2013). Optimization of a cloud resource management problem from a consumer perspective. In D. an Mey, M. Alexander, P. Bientinesi, M. Cannataro, C. Clauss, A. Costan, ... J. Weidendorfer (Eds.), Lecture notes in computer science 8374 (pp. 218-227). Berlin: Springer.

Coutinho, R. d. C., Drummond, L. M. A., Frota, Y., \& de Oliveira, D. (2015). Optimizing virtual machine allocation for parallel scientific workflows in federated clouds. Future Generation Computer Systems, 46, 51-68.

Daniel, W. W. (1990). Applied nonparametric statistics. Boston, MA: PWS-Kent Publishing Company.

Familiar, B. (2015). Microservices, IoT and Azure: Leveraging DevOps and microservice architecture to deliver SaaS Solutions. New York, NY: Apress.

Fujitsu (2014). Performance report primergy CX270S2. Technical Report.

García, S., Molina, D., Lozano, M., \& Herrera, F. (2009). A study on the use of nonparametric tests for analyzing the evolutionary algorithms behaviour: A case study on the cec2005 special session on real parameter optimization. Journal of Heuristics, 15(6), 617-644.

Gartner (2016). Cloud services brokerage (CSB). http://www.gartner.com/it-glossary/ cloud-services-brokerage-csb. Last accessed Dec 08, 2019.

Guo, L., He, Z., Zhao, S., Zhang, N., Wang, J., \& Jiang, C. (2012). Multi-objective optimization for data placement strategy in cloud computing. In C. Liu, L. Wang, \& A. Yang (Eds.), Communications in computer and information science vol. 308 (pp. 119-126). Berlin: Springer.

Gutierrez-Garcia, J. O., \& Sim, K. M. (2012). GA-based cloud resource estimation for agent-based execution of bag-of-tasks applications. Information Systems Frontiers, 14(4), 925-951.

Heilig, L., Lalla-Ruiz, E., \& Voß, S. (2016). A cloud brokerage approach for solving the resource management problem in multi-cloud environments. Computers \& Industrial Engineering, 95, 16-26.

Heilig, L., \& Voß, S. (2014a). Decision analytics for cloud computing: A classification and literature review. In A. Newman, \& J. Leung (Eds.), Tutorials in operations research-bridging data and decisions (pp. 1-26). Catonsville, MD: INFORMS. doi:10.1287/educ.2014.0124.

Heilig, L., \& Voß, S. (2014b). A scientometric analysis of cloud computing literature. IEEE Transactions on Cloud Computing, 2(3), 266-278.

Heilig, L., \& Voß, S. (2017). Managing cloud-based big data platforms: A reference architecture and cost perspective. In F. P. García Márquez, \& B. Lev (Eds.), Big data management (pp. 29-45). Cham: Springer

Heilig. L., Voß, S., \& Wulfken, L. (2015). Building clouds: An integrative approach for an automated deployment of elastic cloud services. In V. Chang, R. Walters, \& G. Wills (Eds.), Delivery and adoption of cloud computing services in contemporary organizations (pp. 269-290). Hershey, PA: IGI.

Hoffa, C., Mehta, G., Freeman, T., Deelman, E., Keahey, K., Berriman, B., \& Good, J. (2008). On the use of cloud computing for scientific workflows. In Proceedings of the 4th ieee fourth international conference on escience (pp. 640-645). IEEE.

IDC (2017). Worldwide semiannual public cloud services spending guide. Technical Report. http://www.idc.com/getdoc.jsp?containerId=prUS42321417. Last accessed March 3, 2018

Khajeh-Hosseini, A., Greenwood, D., Smith, J. W., \& Sommerville, I. (2012). The cloud adoption toolkit: supporting cloud adoption decisions in the enterprise. Software: Practice and Experience, 42(4), 447-465.

KPMG (2017). Journey to the cloud. Technical Report. https://assets.kpmg/content/ dam/kpmg/xx/pdf/2017/02/the-creative-cios-agenda-journey-to-cloud.pdf. Last accessed Dec 08, 2019

de León, A. D., Lalla-Ruiz, E., Melián-Batista, B., \& Moreno-Vega, J. M. (2017). A machine learning-based system for berth scheduling at bulk terminals. Expert Systems with Applications, 87, 170-182.

Liu, F., Tong, J., Mao, J., Bohn, R., Messina, J., Badger, L., \& Leaf, D. (2011). NIST cloud computing reference architecture. Technical Report. National Institute of Standards and Technology.

Lucas-Simarro, J. L., Moreno-Vozmediano, R., Montero, R. S., \& Llorente, I. M. (2011). Dynamic placement of virtual machines for cost optimization in multi-cloud environments. In Proceedings of the international conference on high performance computing and simulation (HPCS) (pp. 1-7). Istanbul: IEEE.

Lucas-Simarro, J. L., Moreno-Vozmediano, R., Montero, R. S., \& Llorente, I. M. (2013). Scheduling strategies for optimal service deployment across multiple clouds. $\mathrm{Fu}$ ture Generation Computer Systems, 29(6), 1431-1441.

Marston, S., Li, Z., Bandyopadhyay, S., Zhang, J., \& Ghalsasi, A. (2011). Cloud computing - the business perspective. Decision Support Systems, 51(1), 176-189. doi:10.1016/j.dss.2010.12.006.

Mauri, G. R., Ribeiro, G. M., Lorena, L. A. N., \& Laporte, G. (2016). An adaptive large neighborhood search for the discrete and continuous berth allocation problem. Computers \& Operations Research, 70, 140-154.

Medernach, E., \& Sanlaville, E. (2012). Fair resource allocation for different scenarios of demands. European Journal of Operational Research, 218(2), 339-350. 
Pandey, S., Wu, L., Guru, S. M., \& Buyya, R. (2010). A particle swarm optimization-based heuristic for scheduling workflow applications in cloud computing environments. In Proceedings of the 24th ieee international conference on advanced information networking and applications (aina) (pp. 400-407). Perth, WA: IEEE.

Pellegrini, P., \& Birattari, M. (2006). The relevance of tuning the parameters of metaheuristics. Technical Report. Technical report, IRIDIA, Université Libre de Bruxelles.

Pisinger, D., \& Ropke, S. (2007). A general heuristic for vehicle routing problems. Computers \& Operations Research, 34(8), 2403-2435.

Pisinger, D., \& Ropke, S. (2010). Large neighborhood search. In M. Gendreau, \& J.-Y. Potvin (Eds.), Handbook of metaheuristics (pp. 399-419). New York, NY: Springer.

Püschel, T., Schryen, G., Hristova, D., \& Neumann, D. (2015). Revenue management for cloud computing providers: decision models for service admission control under non-probabilistic uncertainty. European Journal of Operational Research, 244(2), 637-647.

Ribeiro, G. M., \& Laporte, G. (2012). An adaptive large neighborhood search heuristic for the cumulative capacitated vehicle routing problem. Computers \& Operations Research, 39(3), 728-735.

Ritzinger, U., \& Puchinger, J. (2013). Hybrid metaheuristics for dynamic and stochastic vehicle routing. In E.-G. Talbi (Ed.), Hybrid metaheuristics (pp. 77-95). Heidelberg: Springer.

Rochwerger, B., Breitgand, D., Levy, E., Galis, A., Nagin, K., Llorente, I. M., ... Caceres, J., et al. (2009). The reservoir model and architecture for open federated cloud computing. IBM Journal of Research and Development, 53(4), 1-11.

Ropke, S., \& Pisinger, D. (2006). An adaptive large neighborhood search heuristic for the pickup and delivery problem with time windows. Transportation Science, 40(4), 455-472.
Schrimpf, G., Schneider, J., Stamm-Wilbrandt, H., \& Dueck, G. (2000). Record breaking optimization results using the ruin and recreate principle. Journal of Computational Physics, 159(2), 139-171.

Shaw, P. (1998). Using constraint programming and local search methods to solve vehicle routing problems. In M. Maher, \& J.-F. Puget (Eds.), Principles and practice of constraint programming, lecture notes in computer science 1520 (pp. 417-431). Berlin: Springer.

Shen, S., Deng, K., Iosup, A., \& Epema, D. (2013). Scheduling jobs in the cloud using on-demand and reserved instances. In F. Wolf, B. Mohr, \& D. an Mey (Eds.), Euro-par 2013 parallel processing, lecture notes in computer science 8097 (pp. 242-254). Berlin: Springer.

Subashini, S., \& Kavitha, V. (2011). A survey on security issues in service delivery models of cloud computing. Journal of Network and Computer Applications, 34(1), $1-11$.

Sultan, N., \& van de Bunt-Kokhuis, S. (2012). Organisational culture and cloud computing: coping with a disruptive innovation. Technology Analysis \& Strategic Management, 24(2), 167-179.

Tordsson, J., Montero, R. S., Moreno-Vozmediano, R., \& Llorente, I. M. (2012). Cloud brokering mechanisms for optimized placement of virtual machines across multiple providers. Future Generation Computer Systems, 28(2), 358-367.

Vaquero, L. M., Rodero-Merino, L., Caceres, J., \& Lindner, M. (2009). A break in the clouds: towards a cloud definition. ACM SIGCOMM Computer Communication Review, 39(1), 50-55.

Yu, C., Zhang, D., \& Lau, H. Y. (2017). An adaptive large neighborhood search heuristic for solving a robust gate assignment problem. Expert Systems with Applications, 84, 143-154.

Zissis, D., \& Lekkas, D. (2012). Addressing cloud computing security issues. Future Generation Computer Systems, 28(3), 583-592. 